\title{
INDICES OF VECTOR FIELDS AND 1-FORMS ON SINGULAR VARIETIES
}

\author{
W. EBELING AND S. M. GUSEIN-ZADE
}

\begin{abstract}
We discuss different generalizations of the classical notion of the index of a singular point of a vector field to the case of vector fields or 1-forms on singular varieties, describe relations between them and formulae for their computation.
\end{abstract}

\section{INTRODUCTION}

An isolated singular point (zero) of a (continuous) vector field on a smooth manifold has a well known invariant - the index. A neighbourhood of a point $P$ in a manifold $M^{n}$ of dimension $n$ can be identified with a neighbourhood of the origin in the affine (coordinate) space $\mathbb{R}^{n}$. A germ $X$ of a vector field on $\left(\mathbb{R}^{n}, 0\right)$ can be written as $X(x)=\sum_{i=1}^{n} X_{i}(x) \frac{\partial}{\partial x_{i}}$. Let $B_{\varepsilon}^{n}(0)$ be the ball of radius $\varepsilon$ centred at the origin in $\mathbb{R}^{n}$ such that the vector field $X$ is defined in a neighbourhood of this ball and has no zeros in it except at the origin. Let $S_{\varepsilon}^{n-1}(0)$ be the $(n-1)$-dimensional sphere $\partial B_{\varepsilon}^{n}(0)$. The vector field $X$ defines a map

$$
\frac{X}{\|X\|}: S_{\varepsilon}^{n-1}(0) \rightarrow S_{1}^{n-1}
$$

The index $\operatorname{ind}_{P} X$ of the vector field $X$ at the point $P$ is defined as the degree of the map $\frac{X}{\|X\|}$. One can see that it is independent of the chosen coordinates.

If the vector field $X$ is smooth and its singular point $P$ is non-degenerate, i.e. if $J_{X, P}:=\operatorname{det}\left(\frac{\partial X_{i}}{\partial x_{j}}(0)\right) \neq 0$, then $\operatorname{ind}_{P} X=\operatorname{sgn} J_{X, P}$, i.e. it is equal to 1 if $J_{X, P}>0$ and is equal to -1 if $J_{X, P}<0$. The index of an arbitrary isolated singular point $P$ of a smooth vector field $X$ is equal to the number of non-degenerate singular points which split from the point $P$ under a generic deformation $\widetilde{X}$ of the vector field $X$ in a neighbourhood of the point $P$ counted with the corresponding signs $\left(\operatorname{sgn} J_{\widetilde{X}}, \widetilde{P}\right)$. This follows, in particular, from the fact that the index of a vector field satisfies the law of conservation of number (see below).

This work was partially supported by the DFG-programme "Global methods in complex geometry" (Eb 102/4-3), grants RFBR-04-01-00762, NWO-RFBR 047.011.2004.026. 
One of the most important properties of the index of a vector field is the Poincaré-Hopf theorem. Suppose that the manifold $M$ is closed, i.e. compact without boundary, and that the vector field $X$ has finitely many singular points on it.

Theorem 1 (Poincaré-Hopf). The sum

$$
\sum_{P \in \operatorname{Sing} X} \operatorname{ind}_{P} X
$$

of indices of singular points of the vector field $X$ is equal to the Euler characteristic $\chi(M)$ of the manifold $M$.

If $M$ is an $n$-dimensional complex analytic (compact) manifold, then its Euler characteristic $\chi(M)$ is equal to the characteristic number $\left\langle c_{n}(T M),[M]\right\rangle$, where $c_{n}(M)$ is the top Chern class of the manifold $M$. If a vector field $X$ on a complex manifold $M$ is holomorphic and a singular point $P$ of it is nondegenerate, then the index $\operatorname{ind}_{P} X$ is equal to +1 . The index of an isolated singular point $P$ of a holomorphic vector field $X$ is positive. It is equal to the number of non-degenerate singular points which split from the point $P$ under a generic deformation of the vector field $X$ in a neighbourhood of the point $P$.

There exists an algebraic formula for the index $\operatorname{ind}_{P} X$ of an isolated singular point of a holomorphic vector field. In local coordinates centred at the point $P$, let the vector field $X$ be equal to $\sum_{i=1}^{n} X_{i}(x) \frac{\partial}{\partial x_{i}}$. Let $\mathcal{O}_{\mathbb{C}^{n}, 0}$ be the ring of germs of holomorphic functions of $n$ variables.

\section{Theorem 2.}

$$
\operatorname{ind}_{P} X=\operatorname{dim}_{\mathbb{C}} \mathcal{O}_{\mathbb{C}^{n}, 0} /\left(X_{1}, \ldots, X_{n}\right),
$$

where $\left(X_{1}, \ldots, X_{n}\right)$ is the ideal generated by the germs $X_{1}, \ldots, X_{n}$.

This statement was known for a long time, however, it seems that the first complete proof appeared in [Pa67].

An algebraic formula for the index $\operatorname{ind}_{P} X$ of an isolated (in fact for an algebraically isolated: see the definition below) singular point of a smooth (say, $C^{\infty}$ ) vector field was given in [EL77, Kh77]. In local coordinates centred at the point $P$, let $X=\sum_{i=1}^{n} X_{i}(x) \frac{\partial}{\partial x_{i}}$. Let $\mathcal{E}_{\mathbb{R}^{n}, 0}$ be the ring of germs of smooth $\left(C^{\infty}\right)$ functions of $n$ variables.

Definition: The singular point $P$ of the vector field $v$ is algebraically isolated if the factor $\operatorname{ring} \mathcal{E}_{\mathbb{R}^{n}, 0} /\left(X_{1}, \ldots, X_{n}\right)$ has a finite dimension as a vector space.

If a singular point of a vector field is algebraically isolated, then it is isolated. If, in the local coordinates $x_{1}, \ldots, x_{n}$, the vector field $X$ is (real) analytic (i.e., if the components $X_{i}$ are analytic functions of $x_{1}, \ldots, x_{n}$ ), one can consider its complexification $X_{\mathbb{C}}$ which is a holomorphic vector field in a neighbourhood of the origin in $\mathbb{C}^{n}$. In this case the singular point of 
the vector field $X$ is algebraically isolated if and only if the singular point of the vector field $X_{\mathbb{C}}$ (the origin) is isolated. Let

$$
J_{X}=J_{X}(x):=\operatorname{det}\left(\frac{\partial X_{i}}{\partial x_{j}}\right)
$$

be the Jacobian of the vector field $X$. One can prove that $J_{X} \neq 0$ in the local ring $R_{X}=\mathcal{E}_{\mathbb{R}^{n}, 0} /\left(X_{1}, \ldots, X_{n}\right)$. Moreover, the ring $R_{X}$ has a one dimensional minimal ideal and this ideal is generated by the Jacobian $J_{X}$. Let $\ell: R_{X} \rightarrow \mathbb{R}$ be a linear function on the ring $R_{X}$ (considered as a vector space) such that $\ell\left(J_{X}\right)>0$. Consider the quadratic form $Q$ on $R_{X}$ defined by

$$
Q(\varphi, \psi)=\ell(\varphi \cdot \psi)
$$

Theorem 3 (Eisenbud-Levine-Khimshiashvili). The index $\operatorname{ind}_{P} X$ of the singular point $P$ of the vector field $X$ is equal to the signature $\operatorname{sgn} Q$ of the quadratic form $Q$.

For a proof of this theorem see also AGV85.

In what follows we shall, in particular, discuss generalizations of the notion of the index of a vector field to real or complex analytic variety with singularities and problems of their computation.

\section{1-FORMS VERSUS VECTOR FIELDS}

Traditionally the definition of the index and the corresponding results (say, the Poincaré-Hopf and the Eisenbud-Levine-Khimshiashvili theorems) are formulated for vector fields. However, instead of vector fields one can consider 1-forms. Using a Riemannian metric one can identify vector fields and 1-forms on a smooth $\left(C^{\infty}\right)$ manifold. Therefore on a smooth manifold all notions and statements concerning vector fields can be formulated for 1-forms as well. In particular, the index $\operatorname{ind}_{P} \omega$ of a 1 -form $\omega$ on a smooth $n$-dimensional manifold $M$ at an isolated singular point (zero) $P$ is defined (in local coordinates) as the degree of the map $\frac{\omega}{\|\omega\|}: S_{\varepsilon}^{n-1} \rightarrow S_{1}^{n-1}$, where $S_{1}^{n-1}$ is the unit sphere in the dual space. In the local complex analytic situation one can identify germs of complex analytic vector fields and germs of complex analytic 1-forms using local coordinates: the vector field $\sum X_{i} \frac{\partial}{\partial z_{i}}$ corresponds to the 1 -form $\sum X_{i} d z_{i}$. In the Poincaré-Hopf theorem for 1-forms on an $n$-dimensional complex manifold $M$ the sum of indices of singular points of a 1 -form is equal to $\left\langle c_{n}\left(T^{*} M\right),[M]\right\rangle=(-1)^{n} \chi(M)$. The only essential difference between vector fields and 1-forms in the smooth complex analytic case is that non-trivial complex analytic (global) vector fields and 1-forms exist, generally speaking, on different complex analytic manifolds: in the odd-dimensional case vector fields (respectively 1-forms) can exist only on manifolds with non-negative (respectively non-positive) Euler characteristic.

However, for the case of singular varieties the situation becomes quite different. In a series of papers instead of considering vector fields on a 
variety we started to consider 1-forms. For 1-forms on germs of singular varieties (real or complex analytic) one can define several notions of indices usually corresponding to appropriate analogues for vector fields. However, the properties of the indices for vector fields and for 1 -forms are different. For example, just as above, to an analytic vector field $X=\sum_{i=1}^{N} X_{i} \frac{\partial}{\partial x_{i}}$ on a real variety $(V, 0) \subset\left(\mathbb{R}^{N}, 0\right)$ (or on a complex analytic variety $(V, 0) \subset\left(\mathbb{C}^{N}, 0\right)$ ) one can associate the 1 -form $\omega=\sum_{i=1}^{N} X_{i} d x_{i}$ (dependent on the choice of coordinates $x_{1}, \ldots, x_{N}$ in $\left(\mathbb{R}^{N}, 0\right)$ or in $\left.\left(\mathbb{C}^{N}, 0\right)\right)$. If the vector field $X$ has an isolated singular point on $(V, 0)$, then, for a generic (!) choice of the coordinates $x_{1}, \ldots, x_{N}$, the corresponding 1-form $\omega$ has an isolated singular point as well. This correspondence does not work in the other direction. Moreover, whereas for the radial index on a real analytic variety (see below) the index of a vector field coincides with that of the corresponding 1-form, this does not hold for other indices, say, for the so called GSV index (on an isolated complete intersection singularity: ICIS). In some cases one can say that the notion of an index of a 1-form (say, of a holomorphic one) is somewhat more natural than that of a vector field. For example, the notion of the GSV index of a 1-form (see below) is "more complex analytic" (does not use the complex conjugation for the definition) and "more geometric" (uses only objects of the same tensor type). Moreover the index of an isolated singular point of a holomorphic 1-form on a complex ICIS can be described as the dimension of an appropriate algebra. Finally, on a real ICIS the real index of a 1-form which is the differential of a germ of a function with an algebraically isolated singular point plus-minus the Euler characteristic of a (real) smoothing of the ICIS can be expressed in terms of the signature of a quadratic form on a space (certain space of thimbles), the dimension of which is equal to the (complex) index of the corresponding complexification.

The condition for a vector field to be tangent to a germ of a singular variety is a very restrictive one. For example, holomorphic vector fields with isolated zeros exist on a complex analytic variety with an isolated singularity BG94, but not in general.

Example 1. Consider the surface $X$ in $\mathbb{C}^{3}$ given by the equation

$$
x y(x-y)(x+z y)=0 .
$$

It has singularities on the line $\{x=y=0\}$. It can be considered as a family of four lines in the $(x, y)$-plane with different cross ratios. Then any holomorphic vector field tangent to $X$ vanishes on the line $\{x=y=0\}$, because the translation along such a vector field has to preserve the cross ratio of the lines.

However, 1-forms with isolated singular points always exist. 
The idea to consider indices of 1 -forms instead of indices of vector fields (in some situations) was first formulated by V.I.Arnold (Ar79, see also Ar04).

\section{RADIAL INDEX}

One can say that the notion of the radial index of a vector field or of a 1-form on a singular variety is a straightforward generalization of the usual index inspired by the Poincaré-Hopf theorem.

Let us start with the setting of (real: $C^{\infty}$ ) manifolds with isolated singularities.

A manifold with isolated singularities is a topological space $M$ which has the structure of a smooth (say, $C^{\infty_{-}}$) manifold outside of a discrete set $S$ (the set of singular points of $M$ ). A diffeomorphism between two such manifolds is a homeomorphism which sends the set of singular points onto the set of singular points and is a diffeomorphism outside of them. We say that $M$ has a cone-like singularity at a (singular) point $P \in S$ if there exists a neighbourhood of the point $P$ diffeomorphic to the cone over a smooth manifold $W_{P}\left(W_{P}\right.$ is called the link of the point $P$ ). In what follows we assume all manifolds to have only cone-like singularities. A (smooth or continuous) vector field or 1-form on a manifold $M$ with isolated singularities is a (smooth or continuous) vector field or 1-form respectively on the set $M \backslash S$ of regular points of $M$. The set of singular points $S_{X}$ of a vector field $X$ or the set of singular points $S_{\omega}$ of a 1 -form $\omega$ on a (singular) manifold $M$ is the union of the set of usual singular points of $X$ or of $\omega$ respectively on $M \backslash S$ (i.e., points at which $X$ or $\omega$ respectively tends to zero) and of the set $S$ of singular points of $M$ itself.

For an isolated usual singular point $P$ of a vector field $X$ or of a 1-form $\omega$ there is defined its index $\operatorname{ind}_{P} X$ or $\operatorname{ind}_{P} \omega$ respectively. If the manifold $M$ is closed and has no singularities $(S=\emptyset)$ and the vector field $X$ or the 1-form $\omega$ on $M$ has only isolated singularities, then the sum of these indices over all singular points is equal to the Euler characteristic $\chi(M)$ of the manifold $M$.

Let $(M, P)$ be a cone-like singularity (i.e., a germ of a manifold with such a singular point). Let $X$ be a vector field defined on an open neighbourhood $U$ of the point $P$. Suppose that $X$ has no singular points on $U \backslash\{P\}$. Let $V$ be a closed cone-like neighbourhood of the point $P$ in $U\left(V \cong C W_{P}\right.$, $V \subset U)$. On the cone $C W_{P}=\left(W_{P} \times I\right) /\left(W_{P} \times\{0\}\right)(I=[0,1])$, there is defined a natural vector field $\partial / \partial t(t$ is the coordinate on $I)$. Let $X_{\text {rad }}$ be the corresponding vector field on $V$. Let $\widetilde{X}$ be a (continuous) vector field on $U$ which coincides with $X$ near the boundary $\partial U$ of the neighbourhood $U$ and with $X_{\text {rad }}$ on $V$ and has only isolated singular points. 
Definition: The radial index $\operatorname{ind}_{\mathrm{rad}}(X ; M, P)$ (or simply $\operatorname{ind}_{\mathrm{rad}} X$ ) of the vector field $X$ at the point $P$ is equal to

$$
1+\sum_{\widetilde{P} \in S_{\widetilde{X}} \backslash\{P\}} \operatorname{ind}_{\widetilde{P}} \widetilde{X}
$$

(the sum is over all singular points $\widetilde{P}$ of the vector field $\widetilde{X}$ except $P$ itself).

Analogously, we define the radial index of a 1-form $\omega$ defined on an open neighbourhood $U$ of the point $P$ which has no singular points on $U \backslash\{P\}$. Let $V$ again be a closed cone-like neighbourhood of $P$ in $U\left(V \cong C W_{P}\right.$, $V \subset U)$. On the cone $C W_{P}=\left(W_{P} \times I\right) /\left(W_{P} \times\{0\}\right)(I=[0,1])$ there is defined a natural 1-form $d t$. Let $\omega_{\text {rad }}$ be the corresponding 1-form on $V$. Let $\widetilde{\omega}$ be a (continuous) 1-form on $U$ which coincides with $\omega$ near the boundary $\partial U$ of the neighbourhood $U$ and with $\omega_{\text {rad }}$ on $V$ and has only isolated singular points.

Definition: The radial index $\operatorname{ind}_{\mathrm{rad}}(\omega ; M, P)$ (or simply $\operatorname{ind}_{\mathrm{rad}} \omega$ ) of the 1 -form $\omega$ at the point $P$ is equal to

$$
1+\sum_{\widetilde{P} \in S_{\widetilde{\omega} \backslash\{P\}}} \operatorname{ind}_{\widetilde{P}} \widetilde{\omega}
$$

(the sum is over all singular points $\widetilde{P}$ of the 1-form $\widetilde{\omega}$ except $P$ itself).

For a cone-like singularity at a point $P \in S$, the link $W_{P}$ and thus the cone structure of a neighbourhood are, generally speaking, not well-defined (cones over different manifolds may be locally diffeomorphic). However it is not difficult to show that the indices $\operatorname{ind}_{\mathrm{rad}} X$ and $\operatorname{ind}_{\mathrm{rad}} \omega$ do not depend on the choice of a cone structure on a neighbourhood and on the choice of the vector field $\widetilde{X}$ and the 1 -form $\widetilde{\omega}$ respectively.

Example 2. The index of the radial vector field $X_{\text {rad }}$ and of the radial 1 -form $\omega_{\text {rad }}$ is equal to 1 . The index of the vector field $\left(-X_{\mathrm{rad}}\right)$ and of the 1 -form $\left(-\omega_{\mathrm{rad}}\right)$ is equal to $1-\chi\left(W_{P}\right)$ where $W_{P}$ is the link of the singular point $P$.

Proposition 1. For a vector field $X$ or a 1-form $\omega$ with isolated singular points on a closed manifold $M$ with isolated singularities, the statement of Theorem 1 holds.

Now let $(V, 0) \subset\left(\mathbb{R}^{N}, 0\right)$ be the germ of a real analytic variety, generally speaking, with a non-isolated singular point at the origin. Let $V=\bigcup_{i=1}^{q} V_{i}$ be a Whitney stratification of the germ $(V, 0)$. Let $X$ be a continuous vector field on $(V, 0)$ (i.e., the restriction of a continuous vector field on $\left(\mathbb{R}^{N}, 0\right)$ tangent to $V$ at each point) which has an isolated zero at the origin (on $V$ ). (Tangency to the stratified set $V$ means that for each point $Q \in V$ the vector $X(Q)$ is tangent to the stratum $V_{i}$ which contains the point $Q$.) Let $V_{i}$ be a stratum of dimension $k$ and let $Q$ be a point of $V_{i}$. A neighbourhood of the point $Q$ in $V$ is diffeomorphic to the direct product of a linear space 
$\mathbb{R}^{k}$ and the cone $C W_{Q}$ over a compact singular analytic variety $W_{Q}$. (Here a diffeomorphism between two stratified spaces is a homeomorphism which is a diffeomorphism on each stratum.) Let $\varepsilon>0$ be small enough so that in the closed ball $B_{\varepsilon}$ of radius $\varepsilon$ centred at the origin in $\mathbb{R}^{N}$ the vector field $X$ has no zeros on $V \backslash\{0\}$. It is not difficult to show that there exists a (continuous) vector field $\widetilde{X}$ on $(V, 0)$ such that:

(1) The vector field $\widetilde{X}$ is defined on $V \cap B_{\varepsilon}$.

(2) $\widetilde{X}$ coincides with the vector field $X$ in a neighbourhood of $V \cap S_{\varepsilon}$ in $V \cap B_{\varepsilon}\left(S_{\varepsilon}\right.$ is the sphere $\left.\partial B_{\varepsilon}\right)$.

(3) The vector field $\tilde{X}$ has only a finite number of zeros.

(4) Each point $Q \in U(0)$ with $\widetilde{X}(Q)=0$ has a neighbourhood diffeomorphic to $\left(\mathbb{R}^{k}, 0\right) \times C W_{Q}$ in which $\widetilde{X}(y, z)\left(y \in \mathbb{R}^{k}, z \in C W_{Q}\right)$ is of the form $Y(y)+X_{\operatorname{rad}}(z)$, where $Y$ is a germ of a vector field on $\left(\mathbb{R}^{k}, 0\right)$ with an isolated singular point at the origin, $X_{\text {rad }}$ is the radial vector field on the cone $C W_{Q}$.

Let $S_{\widetilde{X}}$ be the set of zeros of the vector field $\widetilde{X}$ on $V \cap B_{\varepsilon}$. For a point $Q \in S_{\widetilde{X}}$, let $\widetilde{\operatorname{ind}}(Q):=\operatorname{ind}\left(Y ; \mathbb{R}^{k}, 0\right)$, where $Y$ is the vector field on $\left(\mathbb{R}^{k}, 0\right)$ described above. If $k=0$ (this happens at the origin if it is a stratum of the stratification $\left.\left\{V_{i}\right\}\right)$, we set $\widetilde{\operatorname{ind}}(Q):=1$.

Definition: The radial index $\operatorname{ind}_{\mathrm{rad}}(X ; V, 0)$ of the vector field $X$ on the variety $V$ at the origin is the number

$$
\sum_{Q \in S_{\tilde{X}}} \widetilde{\operatorname{ind}}(Q)
$$

One can say that the idea of this definition goes back to M.-H. Schwartz who defined this index for so called radial vector fields Schw65, Schw86a, Schw86b; cf. also KT99. It was used to define characteristic classes for singular varieties, see e.g. the surveys $\mathrm{Br} 00, \mathrm{Se} 02$.

Let $\omega$ be an (arbitrary continuous) 1-form on a neighbourhood of the origin in $\mathbb{R}^{N}$ with an isolated singular point on $(V, 0)$ at the origin. (A point $Q \in V$ is a singular point of the 1-form $\omega$ on $V$ if it is singular for the restriction of the 1 -form $\omega$ to the stratum $V_{i}$ which contains the point $Q$; if the stratum $V_{i}$ is zero-dimensional, the point $Q$ is always singular.) Let $\varepsilon>0$ be small enough so that in the closed ball $B_{\varepsilon}$ of radius $\varepsilon$ centred at the origin in $\mathbb{R}^{N}$ the 1 -form $\omega$ has no singular points on $V \backslash\{0\}$. We say that a 1 -form $\omega$ on a germ $(W, 0)$ is radial if for any analytic curve $\gamma:(\mathbb{R}, 0) \rightarrow(W, 0)$ different from the trivial one $(\gamma(t) \not \equiv 0)$ the value $\omega(\dot{\gamma}(t))$ of the 1-form $\omega$ on the tangent vector to the curve $\gamma$ is positive for positive $t$ small enough. It is easy to see that there exists a 1 -form $\widetilde{\omega}$ on $\mathbb{R}^{N}$ such that:

(1) The 1-form $\widetilde{\omega}$ coincides with the 1-form $\omega$ on a neighbourhood of the sphere $S_{\varepsilon}=\partial B_{\varepsilon}$. 
(2) the vector field $\widetilde{X}$ has only a finite number of zeros;

(3) In a neighbourhood of each singular point $Q \in\left(V \cap B_{\varepsilon}\right) \backslash\{0\}, Q \in V_{i}$, $\operatorname{dim} V_{i}=k$, the 1 -form $\widetilde{\omega}$ looks as follows. There exists a (local) analytic diffeomorphism $h:\left(\mathbb{R}^{N}, \mathbb{R}^{k}, 0\right) \rightarrow\left(\mathbb{R}^{N}, V_{i}, Q\right)$ such that $h^{*} \widetilde{\omega}=\pi_{1}^{*} \widetilde{\omega}_{1}+\pi_{2}^{*} \widetilde{\omega}_{2}$, where $\pi_{1}$ and $\pi_{2}$ are the natural projections $\pi_{1}: \mathbb{R}^{N} \rightarrow \mathbb{R}^{k}$ and $\pi_{2}: \mathbb{R}^{N} \rightarrow \mathbb{R}^{N-k}$ respectively, $\widetilde{\omega}_{1}$ is the germ of a 1 -form on $\left(\mathbb{R}^{k}, 0\right)$ with an isolated singular point at the origin, and $\widetilde{\omega}_{2}$ is a radial 1 -form on $\left(\mathbb{R}^{N-k}, 0\right)$.

Remark. One can demand that the 1-form $\widetilde{\omega}_{1}$ has a non-degenerate singular point (and therefore ind $\left(\widetilde{\omega}_{1}, \mathbb{R}^{k}, 0\right)= \pm 1$ ), however, this is not necessary for the definition.

Let $S_{\widetilde{\omega}}$ be the set of singular points of the 1-form $\widetilde{\omega}$ on $V \cap B_{\varepsilon}$. For a point $Q \in S_{\widetilde{\omega}}$, let $\widetilde{\operatorname{ind}}(Q):=\operatorname{ind}\left(\widetilde{\omega}_{1} ; \mathbb{R}^{k}, 0\right)$. If $k=0$, we set $\widetilde{\operatorname{ind}}(Q):=1$.

Definition: The radial index $\operatorname{ind}_{\text {rad }}(\omega ; V, 0)$ of the 1 -form $\omega$ on the variety $V$ at the origin is the sum

$$
\sum_{Q \in S_{\tilde{\omega}}} \widetilde{\operatorname{ind}}(Q)
$$

One can show that these notions are well defined (for the radial index of a 1-form see [EG05a] ).

Just because of the definition, the (radial) index satisfies the law of conservation of number. For a 1-form this means the following: if a 1-form $\omega^{\prime}$ with isolated singular points on $V$ is close to the 1 -form $\omega$, then

$$
\operatorname{ind}_{\mathrm{rad}}(\omega ; V, 0)=\sum_{Q \in \operatorname{Sing} \omega^{\prime}} \operatorname{ind}_{\mathrm{rad}}\left(\omega^{\prime} ; V, 0\right)
$$

where the sum on the right hand side is over all singular points $Q$ of the 1 -form $\omega^{\prime}$ on $V$ in a neighbourhood of the origin.

The radial index generalizes the usual index for vector fields or 1-forms with isolated singularities on a smooth manifold. In particular one has a generalization of the Poincaré-Hopf theorem:

Theorem 4 (Poincaré-Hopf). For a compact real analytic variety $V$ and a vector field $X$ or a 1-form $\omega$ with isolated singular points on $V$, one has

$$
\sum_{Q} \operatorname{ind}_{\mathrm{rad}}(X ; V, Q)=\sum_{Q} \operatorname{ind}_{\mathrm{rad}}(\omega ; V, Q)=\chi(V)
$$

where $\chi(V)$ denotes the Euler characteristic of the set (variety) $V$.

In the case of radial vector fields, this theorem is due to M.-H. Schwartz Schw65, Schw86a, Schw86b, Schw91, (see also BS81, ASV98, KT99]). For a proof of this theorem for the case of 1-forms see EG05a.

Now let $(V, 0) \subset\left(\mathbb{C}^{N}, 0\right)$ be the germ of a complex analytic variety of pure dimension $n$. Let $\omega$ be a (complex and, generally speaking, continuous) 1form on a neighbourhood of the origin in $\mathbb{C}^{N}$. In fact there is a one-to-one correspondence between complex 1-forms on a complex manifold $M^{n}$ (say, on 
$\mathbb{C}^{N}$ ) and real 1 -forms on it (considered as a real $2 n$-dimensional manifold). Namely, to a complex 1-form $\omega$ one associates the real 1-form $\eta=\operatorname{Re} \omega$; the 1-form $\omega$ can be restored from $\eta$ by the formula $\omega(v)=\eta(v)-i \eta(i v)$ for $v \in T_{x} M^{n}$. This means that the index of the real 1-form Re $\omega$ is an invariant of the complex 1-form $\omega$ itself. However, on a smooth manifold ind $_{M^{n}, x} \operatorname{Re} \omega$ does not coincide with the usual index of the singular point $x$ of the 1 -form $\omega$, but differs from it by the coefficient $(-1)^{n}$. (E.g., the index of the (complex analytic) 1-form $\omega=\sum_{j=1}^{n} x_{j} d x_{j}\left(\left(x_{1}, \ldots, x_{n}\right)\right.$ being the coordinates of $\left.\mathbb{C}^{n}\right)$ is equal to 1 , whence the index of the real 1 -form $\operatorname{Re} \omega=\sum_{j=1}^{n} u_{j} d u_{j}-\sum_{j=1}^{n} v_{j} d v_{j}\left(x_{j}=u_{j}+i v_{j}\right)$ is equal to $(-1)^{n}$.) This explains the following definition.

Definition: The (complex radial) index $\operatorname{ind}_{\mathrm{rad}}^{\mathbb{C}}(\omega ; V, 0)$ of the complex 1form $\omega$ on an $n$-dimensional variety $V$ at the origin is $(-1)^{n}$ times the index of the real 1-form $\operatorname{Re} \omega$ on $V$ :

$$
\operatorname{ind}_{\mathrm{rad}}^{\mathbb{C}}(\omega ; V, 0)=(-1)^{n} \operatorname{ind}_{\mathrm{rad}}(\operatorname{Re} \omega ; V, 0) .
$$

\section{GSV INDEX}

This generalization of the index makes sense for varieties which have isolated complete intersection singularities (in particular, for hypersurfaces with isolated singularities).

Let $V \subset\left(\mathbb{C}^{n+k}, 0\right)$ be an $n$-dimensional isolated complete intersection singularity (ICIS) defined by equations $f_{1}=\ldots=f_{k}=0$ (i.e. $V=f^{-1}(0)$, where $\left.f=\left(f_{1}, \ldots, f_{k}\right):\left(\mathbb{C}^{n+k}, 0\right) \rightarrow\left(\mathbb{C}^{k}, 0\right), \operatorname{dim} V=n\right)$ and let $X=$ $\sum_{i=1}^{n+k} X_{i} \frac{\partial}{\partial z_{i}}$ be a germ of a (continuous) vector field on $\left(\mathbb{C}^{n+k}, 0\right)$ tangent to the ICIS $V$. (The latter means that $X(z) \in T_{z} V$ for all $z \in V \backslash\{0\}$.) Suppose that the vector field $X$ does not vanish on $V$ in a punctured neighbourhood of the origin. In this situation the following index (called the GSV index after X. Gómez-Mont, J. Seade, and A. Verjovsky) is defined.

Let $B_{\varepsilon} \subset \mathbb{C}^{n+k}$ be the ball of radius $\varepsilon$ centred at the origin with (positive) $\varepsilon$ small enough so that all the functions $f_{i}(i=1, \ldots, k)$ and the vector field $X$ are defined in a neighbourhood of $B_{\varepsilon}, V$ is transversal to the sphere $S_{\varepsilon}=\partial B_{\varepsilon}$, and the vector field $X$ has no zeros on $V$ inside the ball $B_{\varepsilon}$ except (possibly) at the origin. Let $K=V \cap S_{\varepsilon}$ be the link of the ICIS $(V, 0)$. The manifold $K$ is $(2 n-1)$-dimensional and has a natural orientation as the boundary of the complex manifold $V \cap B_{\varepsilon} \backslash\{0\}$.

Let $\mathcal{M}(p, q), p \geq q$, be the space of $p \times q$ matrices with complex entries and let $D_{p, q}$ be the subspace of $\mathcal{M}(p, q)$ consisting of matrices of rank less than $q$. The subset $D_{p, q}$ is an irreducible subvariety of $\mathcal{M}(p, q)$ of codimension $p-q+1$. The complement $W_{p, q}=\mathcal{M}(p, q) \backslash D_{p, q}$ is the Stiefel manifold of $q$ frames (collections of $q$ linearly independent vectors) in $\mathbb{C}^{p}$. It is known that the Stiefel manifold $W_{p, q}$ is $2(p-q)$-connected and $H_{2(p-q)+1}\left(W_{p, q}\right) \cong \mathbb{Z}$ (see, 
e.g., $[\mathrm{Hu} 75]$ ). The latter fact also proves that $D_{p, q}$ is irreducible. Since $W_{p, q}$ is the complement of an irreducible complex analytic subvariety of codimension $p-q+1$ in $\mathcal{M}(p, q) \cong \mathbb{C}^{p q}$, there is a natural choice of a generator of the homology group $H_{2(p-q)+1}\left(W_{p, q}\right) \cong \mathbb{Z}$. Namely, the ("positive") generator is the boundary of a small ball in a smooth complex analytic slice transversal to $D_{p, q}$ at a non-singular point. Therefore a map from an oriented smooth $\left(C^{\infty}\right)$ closed $(2(p-q)+1)$-dimensional manifold to $W_{p, q}$ has a degree.

Define the gradient vector field grad $f_{i}$ of a function germ $f_{i}$ by

$$
\operatorname{grad} f_{i}=\left(\overline{\frac{\partial f_{i}}{\partial z_{1}}}, \ldots, \overline{\frac{\partial f_{i}}{\partial z_{n+k}}}\right)
$$

$\left(\operatorname{grad} f_{i}\right.$ depends on the choice of the coordinates $\left.z_{1}, \ldots, z_{n+k}\right)$. One has a map

$$
\Psi=\left(X, \operatorname{grad} f_{1}, \ldots, \operatorname{grad} f_{k}\right): K \rightarrow W_{n+k, k+1}
$$

from the link $K$ to the Stiefel manifold $W_{n+k, k+1}$.

Definition: The $G S V$ index $\operatorname{ind}_{\mathrm{GSV}}(X ; V, 0)$ of the vector field $X$ on the ICIS $V$ at the origin is the degree of the map

$$
\Psi: K \rightarrow W_{n+k, k+1} \text {. }
$$

Remark. Note that one uses the complex conjugation for this definition and the components of the discussed map are of different tensor nature. Whereas $X$ is a vector field, grad $f_{i}$ is more similar to a covector.

This index was first defined in GSV91 for vector fields on isolated hypersurface singularities. In SS96] it was generalized to vector fields on ICIS.

It is convenient to consider the map $\Psi$ as a map from $V$ to $\mathcal{M}(n+k, k+1)$ defined by the formula (10) (in a neighbourhood of the ball $B_{\varepsilon}$ ). It maps the complement of the origin in $V$ to the Stiefel manifold $W_{n+k, k+1}$. This description leads to the following definition of the GSV index.

Proposition 2. The $G S V$ index $\operatorname{ind}_{\mathrm{GSV}}(X ; V, 0)$ of the vector field $X$ on the $I C I S V$ at the origin is equal to the intersection number $\left(\Psi(V) \circ D_{n+k, k+1}\right)$ of the image $\Psi(V)$ of the ICIS $V$ under the map $\Psi$ and the variety $D_{n+k, k+1}$ at the origin.

Note that, even if the vector field $X$ is holomorphic, $\Psi(V)$ is not, generally speaking, a complex analytic variety.

Another definition/description of the GSV index $\operatorname{ind}_{\mathrm{GSV}}(X ; V, 0)$ can be given in the following way. Let $\widetilde{V}=V_{t}=f^{-1}(t) \cap B_{\varepsilon}$, where $t \in \mathbb{C}^{k}, 0<$ $\|t\| \ll \varepsilon$, be the Milnor fibre of the ICIS $V$, i.e. a smoothing of it. For $t$ small enough in a neighbourhood of the sphere $S_{\varepsilon}=\partial B_{\varepsilon}$ the manifolds $V=V_{0}$ and $V_{t}$ "almost coincide". This gives an up to isotopy well defined vector field $\widetilde{X}$ on the manifold $\widetilde{V}$ in a neighbourhood of its boundary $f^{-1}(t) \cap S_{\varepsilon}$ (generally speaking, not a complex analytic one). Let us extend this vector 
field to a vector field (also denoted by $\widetilde{X}$ ) on the entire manifold $\widetilde{V}$ with only isolated zeros.

Proposition 3. One has

$$
\operatorname{ind}_{\mathrm{GSV}}(X ; V, 0)=\sum_{Q \in \operatorname{Sing} \tilde{X}} \operatorname{ind}_{Q} \widetilde{X} .
$$

This definition can be easily generalized to a germ of a complex analytic variety with an isolated singularity and with a fixed smoothing. For example, J. Seade defined in this way an index for a singular point of a vector field on a complex analytic surface with a normal smoothable Gorenstein singularity Se87. For a singularity which is not an ICIS, however, it is possible that such a smoothing does not exist or there may be a number of different smoothings what leads to the situation that the index is not well defined. These difficulties cannot be met for curve singularities. Thus in this situation the corresponding index is well defined (cf. Gor00]). For a general variety with an isolated singularity, this ambiguity can be avoided for germs of functions (i.e. for their differentials in our terms) by defining the corresponding index as a certain residue, what is done in IS03.

Now let $V$ be a compact (say, projective) variety all singular points of which are local ICIS and let $X$ be a vector field on $V$ with isolated singular points. One has the following statement.

Proposition 4. One has

$$
\sum_{Q \in \operatorname{Sing} \omega} \operatorname{ind}_{\mathrm{GSV}}(\omega ; V, Q)=\chi(\widetilde{V}),
$$

where $\widetilde{V}$ is a smoothing of the variety (local complete intersection) $V$.

A similar construction can be considered in the real setting. Namely, let $V=f^{-1}(0) \subset\left(\mathbb{R}^{n+k}, 0\right)$ be a germ of a real $n$-dimensional ICIS, $f=$ $\left(f_{1}, \ldots, f_{k}\right):\left(\mathbb{R}^{n+k}, 0\right) \rightarrow\left(\mathbb{R}^{k}, 0\right)$ is a real analytic map, and let $X=$

$\sum_{i=1}^{n+k} X_{i} d z_{i}$ be a germ of a (continuous) vector field on $\left(\mathbb{R}^{n+k}, 0\right)$ tangent to the variety $V$ outside of the origin. Just as above one defines a map $\Psi$ from the link $K=V \cap S_{\varepsilon}^{n+k-1}$ of the ICIS $(V, 0)$ to the Stiefel manifold $W_{n+k, k+1}^{\mathbb{R}}$ of $(k+1)$-frames in $\mathbb{R}^{n+k}$. The Stiefel manifold $W_{n+k, k+1}^{\mathbb{R}}$ is $(n-2)$-connected and its first non-trivial homology group $H_{n-1}\left(W_{n+k, k+1}^{\mathbb{R}} ; \mathbb{Z}\right)$ is isomorphic to the group $\mathbb{Z}$ of integers for $n$ odd and to the group $\mathbb{Z}_{2}$ of order 2 for $n$ even. Therefore the map $\Psi$ has a degree defined as an integer or as an integer modulo 2 depending on the parity of the dimension $n$. If the manifold $V \backslash\{0\}$ is not connected, the construction can be applied to each connected component of it giving a set of degrees (a multi-degree). This invariant (called real GSV index of the vector field $X$ on the real ICIS $V$ ) was introduced and studied in ASV98. 
In [EG01, EG03a] the notion of the GSV index was adapted to the case of a 1-form. Let $\omega=\sum A_{i} d x_{i}\left(A_{i}=A_{i}(x)\right)$ be a germ of a continuous 1-form on $\left(\mathbb{C}^{n+k}, 0\right)$ which as a 1 -form on the ICIS $V$ has (at most) an isolated singular point at the origin (thus it does not vanish on the tangent space $T_{P} V$ to the variety $V$ at all points $P$ from a punctured neighbourhood of the origin in $V$ ). The 1-forms $\omega, d f_{1}, \ldots, d f_{k}$ are linearly independent for all $P \in K$. Thus one has a map

$$
\Psi=\left(\omega, d f_{1}, \ldots, d f_{k}\right): K \rightarrow W_{n+k, k+1} .
$$

Here $W_{n+k, k+1}$ is the Stiefel manifold of $(k+1)$-frames in the space dual to $\mathbb{C}^{n+k}$.

Definition: The $G S V$ index $\operatorname{ind}_{\mathrm{GSV}}(\omega ; V, 0)$ of the 1 -form $\omega$ on the ICIS $V$ at the origin is the degree of the map

$$
\Psi: K \rightarrow W_{n+k, k+1} \text {. }
$$

Just as above $\Psi$ can be considered as a map from the ICIS $V$ to the space $\mathcal{M}(n+k, k+1)$ of $(n+k) \times(k+1)$ matrices. If the 1 -form $\omega$ is holomorphic, the map $\Psi$ and the set $\Psi(V)$ are complex analytic.

The obvious analogues of Propositions 2, 3, and 4 hold.

There exists an algebraic formula for the index $\operatorname{ind}_{\mathrm{GSV}}(\omega ; V, 0)$ of a holomorphic 1-form $\omega$ on an ICIS $V$ which gives it as the dimension of a certain algebra (see Section 6).

In BSS05a, there was defined a generalization of the notion of the GSV index for a 1-form on a complete intersection singularity $V=f^{-1}(0) \subset$ $\left(\mathbb{C}^{n+k}, 0\right)$ if the map $f=\left(f_{1}, \ldots, f_{k}\right):\left(\mathbb{C}^{n+k}, 0\right) \rightarrow\left(\mathbb{C}^{k}, 0\right)$, defining the singularity satisfies Thom's $a_{f}$ regularity condition. (This condition implies that the Milnor fibre of the complete intersection singularity $V$ is welldefined.)

\section{Homological indeX}

Let $(V, 0) \subset\left(\mathbb{C}^{N}, 0\right)$ be any germ of an analytic variety of pure dimension $n$ with an isolated singular point at the origin. Suppose $X$ is a complex analytic vector field tangent to $(V, 0)$ with an isolated singular point at the origin.

Let $\Omega_{V, 0}^{k}$ be be the module of germs of differentiable $k$-forms on $(V, 0)$, i.e. the factor of the module $\Omega_{\mathbb{C}^{N}, 0}^{k}$ of $k$-forms on $\left(\mathbb{C}^{N}, 0\right)$ by the submodule generated by $f \cdot \Omega_{\mathbb{C}^{N}, 0}^{k}$ and $d f \wedge \Omega_{\mathbb{C}^{N}, 0}^{k}$ for all $f$ from the ideal of functions vanishing on $(V, 0)$. Consider the Koszul complex $\left(\Omega_{V, 0}^{\bullet}, X\right)$ :

$$
0 \longleftarrow \mathcal{O}_{V, 0} \stackrel{X}{\longleftarrow} \Omega_{V, 0}^{1} \stackrel{X}{\longleftarrow} \ldots \stackrel{X}{\longleftarrow} \Omega_{V, 0}^{n} \longleftarrow 0
$$

where the arrows are given by contraction with the vector field $X$. The sheaves $\Omega_{V, 0}^{i}$ are coherent sheaves and the homology groups of the complex 
$\left(\Omega_{V, 0}^{\bullet}, X\right)$ are concentrated at the origin and therefore are finite dimensional. The following definition is due to X. Gómez-Mont Góm98.

Definition: The homological index $\operatorname{ind}_{\text {hom }}(X ; V, 0)=\operatorname{ind}_{\text {hom }} X$ of the vector field $X$ on $(V, 0)$ is the Euler characteristic of the above complex:

$$
\operatorname{ind}_{\text {hom }}(X ; V, 0)=\operatorname{ind}_{\text {hom }} X=\sum_{j=0}^{n}(-1)^{j} h_{j}\left(\Omega_{V, 0}^{\bullet}, X\right),
$$

where $h_{j}\left(\Omega_{V, 0}^{\bullet}, X\right)$ is the dimension of the corresponding homology group as a vector space over $\mathbb{C}$.

The homological index satisfies the law of conservation of number Góm98. Theorem 1.2]. In the case when $V$ is a hypersurface, Gómez-Mont has shown that the homological index is equal to the GSV index.

Given a holomorphic 1-form $\omega$ on $(V, 0)$ with an isolated singularity, we consider the complex $\left(\Omega_{V, 0}^{\bullet}, \wedge \omega\right)$ :

$$
0 \longrightarrow \mathcal{O}_{V, 0} \stackrel{\wedge \omega}{\longrightarrow} \Omega_{V, 0}^{1} \stackrel{\wedge \omega}{\longrightarrow} \ldots \stackrel{\wedge \omega}{\longrightarrow} \Omega_{V, 0}^{n} \longrightarrow 0,
$$

where the arrows are given by the exterior product by the form $\omega$.

This complex is the dual of the Koszul complex considered above. It was used by G.M. Greuel in Gr75 for complete intersections. In EGS04 the definition of Gómez-Mont was adapted to this case.

Definition: The homological index $\operatorname{ind}_{\text {hom }}(\omega ; V, 0)=\operatorname{ind}_{\text {hom }} \omega$ of the 1-form $\omega$ on $(V, 0)$ is $(-1)^{n}$ times the Euler characteristic of the above complex:

$$
\operatorname{ind}_{\text {hom }}(\omega ; V, 0)=\operatorname{ind}_{\text {hom }} \omega=\sum_{j=0}^{n}(-1)^{n-j} h_{j}\left(\Omega_{V, 0}^{\bullet}, \wedge \omega\right),
$$

where $h_{j}\left(\Omega_{V, 0}^{\bullet}, \wedge \omega\right)$ is the dimension of the corresponding homology group as a vector space over $\mathbb{C}$.

In EGS04, there was proved the following statement.

Theorem 5. Let $\omega$ be a holomorphic 1-form on $V$ with an isolated singularity at the origin 0.

(i) If $V$ is smooth, then ind $_{\text {hom }} \omega$ equals the usual index of the holomorphic 1-form $\omega$.

(ii) The homological index satisfies the law of conservation of number: if $\omega^{\prime}$ is a holomorphic 1-form on $V$ close to $\omega$, then:

$$
\operatorname{ind}_{\text {hom }}(\omega ; V, 0)=\operatorname{ind}_{\text {hom }}\left(\omega^{\prime} ; V, 0\right)+\sum \operatorname{ind}_{\text {hom }}\left(\omega^{\prime} ; V, x\right) \text {, }
$$

where the sum on the right hand side is over all those points $x$ in a small punctured neighbourhood of the origin 0 in $V$ where the form $\omega^{\prime}$ vanishes.

(iii) If $(V, 0)$ is an isolated complete intersection singularity, then the homological index ind $_{\text {hom }} \omega$ coincides with the GSV index $\operatorname{ind}_{\mathrm{GSV}} \omega$. 
Statement (ii) follows from GG02.

Remark. We notice that one has an invariant for functions on $(V, 0)$ with an isolated singularity at the origin defined by $f \mapsto \operatorname{ind}_{\text {hom }} d f$. By the theorem above, if $(V, 0)$ is an isolated complete intersection singularity, this invariant counts the number of critical points of the function $f$ on a Milnor fibre of the ICIS $V$.

Remark. Let $(C, 0)$ be a curve singularity and let $(\bar{C}, \overline{0})$ be its normalization. Let $\tau=\operatorname{dim} \operatorname{Ker}\left(\Omega_{\mathrm{C}, 0}^{1} \rightarrow \Omega_{\overline{\mathrm{C}}, \overline{0}}^{1}\right), \lambda=\operatorname{dim} \omega_{C, 0} / c\left(\Omega_{C, 0}^{1}\right)$, where $\omega_{C, 0}$ is the dualizing module of Grothendieck, $c: \Omega_{C, 0}^{1} \rightarrow \omega_{C, 0}$ is the class map (see BG80]). In MvS01 there is considered a Milnor number of a function $f$ on a curve singularity introduced by V.Goryunov Gor00. One can see that this Milnor number can be defined for a 1-form $\omega$ with an isolated singularity on $(C, 0)$ as well (as $\operatorname{dim} \omega_{C, 0} / \omega \wedge \mathcal{O}_{C, 0}$ ) and is equal to ind $\mathrm{hom}_{\mathrm{hom}} \omega+\lambda-\tau$.

The laws of conservation of numbers for the homological and the radial indices of 1-forms together with the fact that these two indices coincide on smooth varieties imply that their difference is a locally constant, and therefore constant, function on the space of 1-forms on $V$ with isolated singularities at the origin. Therefore one has the following statement.

Proposition 5. Let $(V, 0)$ be a germ of a complex analytic space of pure dimension $n$ with an isolated singular point at the origin. Then the difference

$$
\operatorname{ind}_{\text {hom }} \omega-\operatorname{ind}_{\text {rad }} \omega
$$

between the homological and the radial index does not depend on the 1-form $\omega$.

If $(V, 0)$ is an ICIS, this difference is equal to the Milnor number of $(V, 0)$. Together with this fact proposition 5 permits to consider the difference

$$
\nu(V, 0):=\operatorname{ind}_{\text {hom }}(\omega ; V, 0)-\operatorname{ind}_{\text {rad }}(\omega ; V, 0)
$$

as a generalized Milnor number of the singularity $(V, 0)$.

There are other invariants of isolated singularities of complex analytic varieties which coincide with the Milnor number for isolated complete intersection singularities. One of them is $(-1)^{n}$ times the reduced Euler characteristic (i.e., the Euler characteristic minus 1) of the absolute de Rham complex of $(V, 0)$ :

$$
\begin{gathered}
0 \longrightarrow \mathcal{O}_{V, 0} \stackrel{d}{\longrightarrow} \Omega_{V, 0}^{1} \stackrel{d}{\longrightarrow} \ldots \stackrel{d}{\longrightarrow} \Omega_{V, 0}^{n} \longrightarrow 0: \\
\bar{\chi}(V, 0):=\sum_{i=0}^{n}(-1)^{n-i} h_{i}\left(\Omega_{V, 0}^{\bullet}, d\right)-(-1)^{n} .
\end{gathered}
$$

In EGS04] it was shown that: 
Theorem 6. One has

$$
\nu(V, 0)=\bar{\chi}(V, 0)
$$

if

(i) $(V, 0)$ is a curve singularity,

(ii) $(V, 0) \subset\left(\mathbb{C}^{d+1}, 0\right)$ is the cone over the rational normal curve in $\mathbb{C P}^{d}$.

Statement (i) implies that the invariant $\nu(V, 0)$ is different from the Milnor number introduced by R.-O. Buchweitz and G.-M. Greuel BG80 for curve singularities. Statement (ii) was obtained with the help of H.-Ch. Graf von Bothmer and R.-O. Buchweitz. For $d=4$ this is Pinkham's example Pi74] of a singularity which has smoothings with different Euler characteristics.

\section{EULER OBSTRUCTION}

The idea of the Euler obstruction emerged from Mac74 where the Euler obstruction of a singular point of a complex analytic variety was defined (the definition was formulated in terms of obstruction theory in [BS81]).

The Euler obstruction of an isolated singular point (zero) of a vector field on a (singular) complex analytic variety was essentially defined in BMPS04 (though formally speaking there it was defined only for holomorphic functions, i.e. for corresponding gradient vector fields). The main result of BMPS04 (see Theorem[7below) gives a relation between the Euler obstruction of a function germ on a singular variety $V$ and the Euler obstructions of singular points of $V$ itself.

In BMPS04, there is introduced the notion of the local Euler obstruction of a holomorphic function with an isolated critical point on the germ of a complex analytic variety. It is defined as follows.

Let $(V, 0) \subset\left(\mathbb{C}^{N}, 0\right)$ be the germ of a purely $n$-dimensional complex analytic variety with a Whitney stratification $V=\bigcup_{i=1}^{q} V_{i}$ and let $f$ be a holomorphic function defined in a neighbourhood of the origin in $\mathbb{C}^{N}$ with an isolated singular point on $V$ at the origin. Let $\varepsilon>0$ be small enough such that the function $f$ has no singular points on $V \backslash\{0\}$ inside the ball $B_{\varepsilon}$. Let $\operatorname{grad} f$ be the gradient vector field of $f$ as defined in Section 3 . Since $f$ has no singular points on $V \backslash\{0\}$ inside the ball $B_{\varepsilon}$, the angle of $\operatorname{grad} f(x)$ and the tangent space $T_{x} V_{i}$ to a point $x \in V_{i} \backslash\{0\}$ is less than $\pi / 2$. Denote by $\zeta_{i}(x) \neq 0$ the projection of $\operatorname{grad} f(x)$ to the tangent space $T_{x} V_{i}$.

Following the construction in BMPS04, the vector fields $\zeta_{i}$ can be glued together to obtain a stratified vector field $\operatorname{grad}_{V} f$ on $V$ such that $\operatorname{grad}_{V} f$

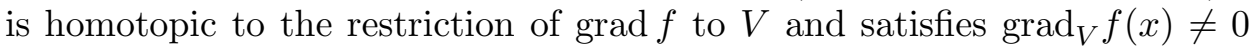
unless $x=0$.

Let $\nu: \widehat{V} \rightarrow V$ be the Nash transformation of the variety $V$ defined as follows. Let $G(n, N)$ be the Grassmann manifold of $n$-dimensional vector subspaces of $\mathbb{C}^{N}$. For a suitable neighbourhood $U$ of the origin in $\mathbb{C}^{N}$, there is a natural map $\sigma: V_{\text {reg }} \cap U \rightarrow U \times G(n, N)$ which sends a point $x$ to $\left(x, T_{x} V_{\text {reg }}\right)$ ( $V_{\text {reg }}$ is the non-singular part of $\left.V\right)$. The Nash transform $\widehat{V}$ is 
the closure of the image $\operatorname{Im} \sigma$ of the map $\sigma$ in $U \times G(n, N)$. The Nash bundle $\widehat{T}$ over $\widehat{V}$ is a vector bundle of rank $n$ which is the pullback of the tautological bundle on the Grassmann manifold $G(n, N)$. There is a natural lifting of the Nash transformation to a bundle map from the Nash bundle $\widehat{T}$ to the restriction of the tangent bundle $T \mathbb{C}^{N}$ of $\mathbb{C}^{N}$ to $V$. This is an isomorphism of $\widehat{T}$ and $T V_{\text {reg }} \subset T \mathbb{C}^{N}$ over the regular part $V_{\text {reg }}$ of $V$.

The vector field $\operatorname{grad}_{V} f$ gives rise to a section $\widehat{\zeta}$ of the Nash bundle $\widehat{T}$ over the Nash transform $\widehat{V}$ without zeros outside of the preimage of the origin.

Definition: BMPS04] The local Euler obstruction $\mathrm{Eu}_{V, 0} f$ of the function $f$ on $V$ at the origin is the obstruction to extend the non-zero section $\widehat{\zeta}$ from the preimage of a neighbourhood of the sphere $S_{\varepsilon}=\partial B_{\varepsilon}$ to the preimage of its interior, more precisely its value (as an element of $H^{2 n}\left(\nu^{-1}\left(V \cap B_{\varepsilon}\right), \nu^{-1}(V \cap\right.$ $\left.\left.S_{\varepsilon}\right)\right)$ ) on the fundamental class of the pair $\left(\nu^{-1}\left(V \cap B_{\varepsilon}\right), \nu^{-1}\left(V \cap S_{\varepsilon}\right)\right)$.

The word local will usually be omitted.

Remark. The local Euler obstruction can also be defined for the restriction to $V$ of a real analytic function on $\mathbb{R}^{2 N}$. In particular, one can take for $f$ the squared distance on $V$ to the origin. In this case, the invariant $\mathrm{Eu}_{V, 0} f$ is the usual local Euler obstruction $\operatorname{Eu}_{V}(0)$ of the variety $(V, 0)$ defined in Mac74, BS81, BLS00].

Denote by $M_{f}=M_{f, t_{0}}$ the Milnor fibre of $f$, i.e. the intersection $V \cap B_{\varepsilon} \cap$ $f^{-1}\left(t_{0}\right)$ for a regular value $t_{0}$ of $f$ close to 0 . In BMPS04, Theorem 3.1] the following result is proved.

Theorem 7. Let $f:(V, 0) \rightarrow(\mathbb{C}, 0)$ have an isolated singularity at $0 \in V$. Then

$$
\operatorname{Eu}_{V}(0)=\left(\sum_{i=1}^{q} \chi\left(M_{f} \cap V_{i}\right) \cdot \operatorname{Eu}_{V}\left(V_{i}\right)\right)+\operatorname{Eu}_{V, 0} f,
$$

where $\operatorname{Eu}_{V}\left(V_{i}\right)$ is the value of the Euler obstruction of $V$ at any point of $V_{i}$, $i=1, \ldots, q$.

One can also define the local Euler obstruction of a stratified vector field on a germ of a complex analytic variety $(V, 0)$ with a Whitney stratification BSS05b. Let $V_{i}$ be a stratum of the Whitney stratification with $0 \in V_{i}$ and $X_{i}$ be a vector field on $V_{i}$ with an isolated singularity at 0 . The Proportionality Theorem of [BS81, BSS05b] states that the local Euler obstruction of a radial extension $X$ of $X_{i}$ at 0 is equal to the local Euler obstruction $\operatorname{Eu}_{V}(0)$ of $(V, 0)$ times the radial index $\operatorname{ind}_{\text {rad }}(X ; V, 0)$ of $X$.

In EG05a the definition of the local Euler obstruction of a function was adapted to the case of a 1-form. Let $\omega$ be a 1-form on a neighbourhood of the origin in $\mathbb{C}^{N}$ with an isolated singular point on $V$ at the origin. Let $\varepsilon>0$ be small enough such that the 1-form $\omega$ has no singular points on $V \backslash\{0\}$ inside the ball $B_{\varepsilon}$.

The 1-form $\omega$ gives rise to a section $\widehat{\omega}$ of the dual Nash bundle $\widehat{T}^{*}$ over the Nash transform $\widehat{V}$ without zeros outside of the preimage of the origin. 
Definition: The local Euler obstruction $\mathrm{Eu}_{V, 0} \omega$ of the 1-form $\omega$ on $V$ at the origin is the obstruction to extend the non-zero section $\widehat{\omega}$ from the preimage of a neighbourhood of the sphere $S_{\varepsilon}=\partial B_{\varepsilon}$ to the preimage of its interior, more precisely its value (as an element of the cohomology group $\left.H^{2 n}\left(\nu^{-1}\left(V \cap B_{\varepsilon}\right), \nu^{-1}\left(V \cap S_{\varepsilon}\right)\right)\right)$ on the fundamental class of the pair $\left(\nu^{-1}\left(V \cap B_{\varepsilon}\right), \nu^{-1}\left(V \cap S_{\varepsilon}\right)\right)$.

Remark. The local Euler obstruction can also be defined for a real 1-form on the germ of a real analytic variety if the last one is orientable in an appropriate sense.

Example 3. Let $\omega=d f$ for the germ $f$ of a holomorphic function on $\left(\mathbb{C}^{N}, 0\right)$. Then $\mathrm{Eu}_{V, 0} d f$ differs from the Euler obstruction $\mathrm{Eu}_{f, V}(0)$ of the function $f$ by the sign $(-1)^{n}$. The reason is that for the germ of a holomorphic function with an isolated critical point on $\left(\mathbb{C}^{n}, 0\right)$ one has $\operatorname{Eu}_{f, V}(0)=$ $(-1)^{n} \mu_{f}$ (see BMPS04, Remark 3.4]), whence $\operatorname{Eu}_{V, 0} d f=\mu_{f}\left(\mu_{f}\right.$ is the Milnor number of the germ $f$ ). E.g., for $f\left(x_{1}, \ldots, x_{n}\right)=x_{1}^{2}+\ldots+x_{n}^{2}$ the obstruction $\operatorname{Eu}_{f, V}(0)$ is the index of the vector field $\sum_{i=1}^{n} \bar{x}_{i} \partial / \partial x_{i}$ (which is equal to $\left.(-1)^{n}\right)$, but the obstruction $\mathrm{Eu}_{V, 0} d f$ is the index of the (holomorphic) 1 -form $\sum_{i=1}^{n} x_{i} d x_{i}$ which is equal to 1 .

The Euler obstruction of a 1-form satisfies the law of conservation of number (just as the radial index). Moreover, on a smooth variety the Euler obstruction and the radial index coincide. This implies the following statement (cf. Theorem [7). We set $\bar{\chi}(Z):=\chi(Z)-1$ and call it the reduced (modulo a point) Euler characteristic of the topological space $Z$ (though, strictly speaking, this name is only correct for a non-empty space $Z$ ).

Proposition 6. Let $(V, 0) \subset\left(\mathbb{C}^{N}, 0\right)$ have an isolated singular point at the origin and let $\ell: \mathbb{C}^{N} \rightarrow \mathbb{C}$ be a generic linear function. Then

$$
\operatorname{ind}_{\mathrm{rad}}(\omega ; V, 0)-\mathrm{Eu}_{V, 0} \omega=\operatorname{ind}_{\mathrm{rad}}(d \ell ; V, 0)=(-1)^{n-1} \bar{\chi}\left(M_{\ell}\right),
$$

where $M_{\ell}$ is the Milnor fibre of the linear function $\ell$ on $V$. In particular

$$
\mathrm{Eu}_{V, 0} d f=(-1)^{n}\left(\chi\left(M_{\ell}\right)-\chi\left(M_{f}\right)\right) .
$$

An analogue of the Proportionality Theorem for 1-forms was proved in BSS05a.

Now let $(V, 0) \subset\left(\mathbb{C}^{N}, 0\right)$ be an arbitrary germ of an analytic variety with a Whitney stratification $V=\bigcup_{i=0}^{q} V_{i}$, where we suppose that $V_{0}=\{0\}$. For a stratum $V_{i}, i=0, \ldots, q$, let $N_{i}$ be the normal slice in the variety $V$ to the stratum $V_{i}\left(\operatorname{dim} N_{i}=\operatorname{dim} V-\operatorname{dim} V_{i}\right)$ at a point of the stratum $V_{i}$ and let $n_{i}$ be the index of a generic (non-vanishing) 1-form $d \ell$ on $N_{i}$ :

$$
n_{i}=(-1)^{\operatorname{dim} N_{i}-1} \bar{\chi}\left(M_{\left.\ell\right|_{N_{i}}}\right) \text {. }
$$

In particular for an open stratum $V_{i}$ of $V, N_{i}$ is a point and $n_{i}=1$. The strata $V_{i}$ of $V$ are partially ordered: $V_{i} \prec V_{j}$ (we shall write $i \prec j$ ) iff $V_{i} \subset \overline{V_{j}}$ and $V_{i} \neq V_{j} ; i \preceq j$ iff $i \prec j$ or $i=j$. For two strata $V_{i}$ and $V_{j}$ 
with $i \preceq j$, let $N_{i j}$ be the normal slice of the variety $\overline{V_{j}}$ to the stratum $V_{i}$ at a point of it $\left(\operatorname{dim} N_{i j}=\operatorname{dim} V_{j}-\operatorname{dim} V_{i}, N_{i i}\right.$ is a point) and let $n_{i j}$ be the index of a generic 1-form $d \ell$ on $N_{i j}$ : $n_{i j}=(-1)^{\operatorname{dim} N_{i j}-1} \bar{\chi}\left(M_{\left.\ell\right|_{N_{i j}}}\right)$, $n_{i i}=1$. Let us define the Euler obstruction $\operatorname{Eu}_{Y, 0} \omega$ to be equal to 1 for a zero-dimensional variety $Y$ (in particular $\operatorname{Eu}_{\overline{V_{0}}, 0} \omega=1, \operatorname{Eu}_{N_{i i}, 0} \omega=1$ ). In EG05a, the following statement was proved.

Theorem 8. One has

$$
\operatorname{ind}_{\mathrm{rad}}(\omega ; V, 0)=\sum_{i=0}^{q} n_{i} \cdot \operatorname{Eu}_{\overline{V_{i}}, 0} \omega .
$$

To write an "inverse" of the formula of Theorem 8. suppose that the variety $V$ is irreducible and $V=\overline{V_{q}}$. (Otherwise one can permit $V$ to be reducible, but also permit the open stratum $V_{q}$ to be not connected and dense; this does not change anything in Theorem 8) Let $m_{i j}$ be the (Möbius) inverse of the function $n_{i j}$ on the partially ordered set of strata, i.e.

$$
\sum_{i \preceq j \preceq k} n_{i j} m_{j k}=\delta_{i k}
$$

For $i \prec j$ one has

$$
\begin{aligned}
m_{i j} & =\sum_{i=k_{0} \prec k_{1} \prec \ldots \prec k_{r}=j}(-1)^{r} n_{k_{0} k_{1}} n_{k_{1} k_{2}} \ldots n_{k_{r-1} k_{r}} \\
& =(-1)^{\operatorname{dim} V-\operatorname{dim} V_{i}} \sum_{i=k_{0} \prec \ldots \prec k_{r}=j} \bar{\chi}\left(M_{\ell_{N_{k_{0} k_{1}}}}\right) \cdot \ldots \cdot \bar{\chi}\left(M_{\ell_{\left.\right|_{k_{k_{r-1}} k_{r}}}}\right) .
\end{aligned}
$$

Corollary 1. One has

$$
\operatorname{Eu}_{V, 0} \omega=\sum_{i=0}^{q} m_{i q} \cdot \operatorname{ind}_{\mathrm{rad}}\left(\omega ; \overline{V_{i}}, 0\right) .
$$

In particular

$$
\begin{aligned}
& \operatorname{Eu}_{V, 0} d f=(-1)^{\operatorname{dim} V-1} \times \\
& \quad\left(\bar{\chi}\left(M_{\left.f\right|_{V}}\right)+\sum_{i=0}^{q-1} \bar{\chi}\left(M_{\left.f\right|_{\overline{V_{i}}}}\right) \sum_{i=k_{0} \prec \ldots \prec k_{r}=q} \bar{\chi}\left(M_{\left.\ell\right|_{N_{k_{0} k_{1}}}}\right) \ldots \bar{\chi}\left(M_{\left.\ell\right|_{N_{k_{r}-1} k_{r}}}\right)\right) .
\end{aligned}
$$

In [T81] the local Euler obstruction of a germ of a variety is related to polar invariants of the germ. A global version of the Euler obstruction of a variety and its connections with global polar invariants are discussed in STV05a.

\section{Algebraic, Analytic, And topological Formulae for indices}

In the introduction, there were mentioned algebraic formulae for the index of an analytic vector field or a 1-form on a smooth manifold: as the 
dimension of a ring in the complex setting and as the signature of a quadratic form in the real one. It is natural to try to look for analogues of such formulae for vector fields or 1-forms on singular varieties. This appeared to be a rather complicated problem. Other sorts of formulae: analytic (usually as certain residues) or topological ones are of interest as well.

A substantial progress in this direction was achieved for the GSV index.

Let $(V, 0)$ be the germ of a hypersurface in $\left(\mathbb{C}^{n+1}, 0\right)$ defined by a germ of a holomorphic function $f:\left(\mathbb{C}^{n+1}, 0\right) \rightarrow(\mathbb{C}, 0)$ with an isolated singular point at 0 . Let

$$
X=\sum_{i=1}^{n+1} X_{i} \frac{\partial}{\partial x_{i}}
$$

be a holomorphic vector field on $\mathbb{C}^{n+1}$ tangent to $V$ with an isolated (in $\left.\mathbb{C}^{n+1}, 0\right)$ zero at the origin. This implies that $X f=h f$ for some $h \in \mathcal{O}_{\mathbb{C}^{n+1}, 0}$. Define the following ideals in $\mathcal{O}_{\mathbb{C}^{n+1}, 0}$ :

$$
\begin{aligned}
J_{f} & :=\left(f_{1}, \ldots, f_{n+1}\right), \\
J_{X} & :=\left(X_{1}, \ldots, X_{n+1}\right), \\
J_{1} & :=\left(h, J_{X}\right), \\
J_{2} & :=\left(f, J_{f}\right), \\
J_{3} & :=\left(f, J_{X}\right) .
\end{aligned}
$$

X. Gómez-Mont Góm98 has proved the following formula for the GSV index:

Theorem 9. For a vector field $X$ with an isolated zero in the ambient space tangent to the germ of a hypersurface $(V, 0)$ with an isolated singularity at 0 , we have: for even $n$

$$
\operatorname{Ind}_{\mathrm{GSV}}(X ; V, 0)=\operatorname{dim} \mathcal{O}_{\mathbb{C}^{n+1}, 0} / J_{X}+\operatorname{dim} \mathcal{O}_{\mathbb{C}^{n+1}, 0} / J_{1}+\operatorname{dim} \mathcal{O}_{\mathbb{C}^{n+1}, 0} / J_{2},
$$

for $n$ odd

$$
\operatorname{Ind}_{\mathrm{GSV}}(X ; V, 0)=\operatorname{dim} \mathcal{O}_{\mathbb{C}^{n+1}, 0} / J_{2}+\operatorname{dim} \mathcal{O}_{\mathbb{C}^{n+1}, 0} / J_{3} .
$$

In this case the GSV index coincides with the homological index. O. Klehn K103 generalized this formula for the homological index to the case when the vector field has an isolated zero on the hypersurface singularity $(V, 0)$ but not necessarily in $\left(\mathbb{C}^{N}, 0\right)$. Recently, H.-Ch. Graf von Bothmer, X. GómezMont and the first author gave formulae to compute the homological index in the case when $V$ is a complete intersection BEG05.

In GM97, GM99 algebraic formulae for the index of a real analytic vector field with an algebraically isolated singular point at the origin tangent to a real hypersurface with an algebraically isolated singularity at 0 are derived. The index is expressed as the signature of a certain non-degenerate quadratic form for an even-dimensional hypersurface and as the difference of the signatures of two non-degenerate quadratic forms in the odd-dimensional case. 
O. Klehn K105] proved that the GSV index coincides with the dimension of a certain explicitly constructed vector space in the case when $(V, 0)$ is an isolated complete intersection singularity of dimension 1 and $X$ is a vector field tangent to $V$ with an isolated zero on $V$ which is deformable in a certain way. He also obtained a signature formula for the real GSV index in the corresponding real analytic case generalizing the Eisenbud-LevineKhimshiashvili formula.

Let $(V, 0) \subset\left(\mathbb{C}^{n+k}, 0\right)$ be an isolated complete intersection singularity defined by an analytic map

$$
f=\left(f_{1}, \ldots, f_{k}\right):\left(\mathbb{C}^{n+k}, 0\right) \rightarrow\left(\mathbb{C}^{k}, 0\right) .
$$

Let $X$ be a holomorphic vector field on $\mathbb{C}^{n+k}$ defined in a suitable neighbourhood $U$ of the origin, tangent to $V$, with an isolated singular point at the origin. Let $C$ be the $k \times k$ matrix whose entries are holomorphic functions on $U$ such that $X \cdot f=C f$. Assume that $\left(x_{1}, \ldots, x_{n+k}\right)$ is a system of coordinates on $U$ such that when $X$ is written as

$$
X=\sum_{i=1}^{n+k} X_{i} \frac{\partial}{\partial x_{i}},
$$

the sequence $\left(X_{1}, \ldots, X_{n}, f_{1}, \ldots, f_{k}\right)$ is regular. Let $J$ denote the Jacobian matrix

$$
J=\left(\frac{\partial X_{i}}{\partial x_{j}}\right) .
$$

We denote by $c_{q}$ the coefficient at $t^{q}$ in the formal power series expansion of

$$
\operatorname{det}\left(I_{n+k}-t \frac{\sqrt{-1}}{2 \pi} J\right)\left[\operatorname{det}\left(I_{k}-t \frac{\sqrt{-1}}{2 \pi} C\right)\right]^{-1}
$$

in $t$, where $I_{n+k}$ and $I_{k}$ denote the identity matrices of sizes $n+k$ and $k$ respectively. In LSS95] the following formula for the GSV index is proved.

Theorem 10. Let $\varepsilon$ be small enough such that the real hypersurfaces $\left|X_{i}\right|=$ $\varepsilon$ are in general position and let $Z$ be the set $\left\{f=0,\left|X_{i}\right|=\varepsilon, 1 \leq i \leq n\right\}$. We assume that $Z$ is oriented so that

$$
d\left(\arg X_{1}\right) \wedge d\left(\arg X_{2}\right) \wedge \ldots \wedge d\left(\arg X_{n}\right)
$$

is positive. Then

$$
\operatorname{Ind}_{\mathrm{GSV}}(X ; V, 0)=\int_{Z} \frac{c_{n} d x_{1} \wedge \ldots \wedge d x_{n}}{\prod_{i=1}^{n} X_{i}}
$$

For generalizations of Theorem 10 and related results see LS95, Su95, Su98, Su02, IS03.

Now let $\omega$ be a holomorphic 1 -form on the ICIS $(V, 0)=f^{-1}(0) \subset$ $\left(\mathbb{C}^{n+k}, 0\right), f=\left(f_{1}, \ldots, f_{k}\right):\left(\mathbb{C}^{n+k}, 0\right) \rightarrow\left(\mathbb{C}^{k}, 0\right)$, i.e. the restriction to 
$(V, 0)$ of a holomorphic 1-form

$$
\omega=\sum_{i=1}^{n+k} A_{i}(x) d x_{i}
$$

on $\left(\mathbb{C}^{n+k}, 0\right)$. Assume that $\omega$ has an isolated singular point at the origin (on $(V, 0))$. Let $I$ is the ideal generated by $f_{1}, \ldots, f_{k}$ and the $(k+1) \times(k+1)$ minors of the matrix

$$
\left(\begin{array}{ccc}
\frac{\partial f_{1}}{\partial x_{1}} & \cdots & \frac{\partial f_{1}}{\partial x_{n+k}} \\
\vdots & \cdots & \vdots \\
\frac{\partial f_{k}}{\partial x_{1}} & \cdots & \frac{\partial f_{k}}{\partial x_{n+k}} \\
A_{1} & \cdots & A_{n+k}
\end{array}\right)
$$

Then one has the following formula for the GSV index EG01, EG03a, EG03b.

Theorem 11. One has

$$
\operatorname{ind}_{\mathrm{GSV}}(\omega ; V, 0)=\operatorname{dim} \mathcal{O}_{\mathbb{C}^{n+k}, 0} / I .
$$

(Note that there is a minor mistake in the proof of this theorem in EG03a. which is corrected in EG05d].) This formula was obtained by Lê D.T. and G.-M. Greuel for the case when $\omega$ is the differential of a function (Gr75, Lê74).

T. Gaffney Ga05 described connections between the GSV index of a holomorphic 1-form on an ICIS and the multiplicity of pairs of certain modules.

In EG05c, there was constructed a quadratic form on the algebra $\mathcal{A}:=$ $\mathcal{O}_{\mathbb{C}^{n+k}, 0} / I$ generalizing the Eisenbud-Levine-Khimshiashvili quadratic form defined for a smooth $V$. This is defined as follows.

Let $F:\left(\mathbb{C}^{n+k} \times \mathbb{C}_{\varepsilon}^{M}, 0\right) \rightarrow\left(\mathbb{C}^{k} \times \mathbb{C}_{\varepsilon}^{M}, 0\right)$ be a deformation of the map $f:\left(\mathbb{C}^{n+k}, 0\right) \rightarrow\left(\mathbb{C}^{k}, 0\right)\left(F(x, \varepsilon)=\left(f_{\varepsilon}(x), \varepsilon\right), f_{0}=f, f_{\varepsilon}=\left(f_{1 \varepsilon}, \ldots, f_{k \varepsilon}\right)\right)$ and let $\omega_{\varepsilon}$ be a deformation of the form $\omega$ (defined in a neighbourhood of the origin in $\left.\mathbb{C}^{n+k} \times \mathbb{C}_{\varepsilon}^{M}\right)$ such that, for generic $\varepsilon \in\left(\mathbb{C}_{\varepsilon}^{M}, 0\right)$, the preimage $f_{\varepsilon}^{-1}(0)$ is smooth and (the restriction of) the form $\omega_{\varepsilon}$ to it has only non degenerate singular points.

Let $\Sigma \subset\left(\mathbb{C}_{\varepsilon}^{M}, 0\right)$ be the germ of the set of the values of the parameters $\varepsilon$ from $\left(\mathbb{C}_{\varepsilon}^{M}, 0\right)$ such that either the preimage $f_{\varepsilon}^{-1}(0)$ is singular or the restriction of the 1 -form $\omega_{\varepsilon}$ to it has degenerate singular points.

Let $\sum_{i=1}^{n} A_{i} d y^{i}$ be a 1 -form on a smooth complex analytic manifold of dimension $n$ (in local coordinates $y^{1}, \ldots, y^{n}$ ). At points $P$ where the 1 -form $\omega$ vanishes, the Jacobian matrix $\mathcal{J}=\left(\frac{\partial A_{i}}{\partial y^{j}}\right)$ defines the tensor $\sum_{i, j} \frac{\partial A_{i}}{\partial y^{j}} d y^{i} \otimes d y^{j}$ of type $(0,2)$ (i.e. a bilinear form on the tangent space). The determinant $J$ of the matrix $\mathcal{J}=\left(\frac{\partial A_{i}}{\partial y^{j}}\right)$ is not a scalar (it depends on the choice of local 
coordinates). Under a change of coordinates it is multiplied by the square of the Jacobian of the change of coordinates (since the Jacobian matrix $\mathcal{J}$ is transformed to $C^{T} \mathcal{J} C$ ). Therefore it should be considered as the coefficient in the tensor

$$
J\left(d y^{1} \wedge \ldots \wedge d y^{m}\right)^{\otimes 2}
$$

of type $(0,2 m)$. In this sense the Jacobian of a 1-form is a sort of a "quadratic differential". To get a number $\widetilde{J}(P)$, one can divide this tensor by the tensor square of a volume form.

Let us fix volume forms on $\mathbb{C}^{n+k}$ and $\mathbb{C}^{k}$, say, the standard ones $\sigma_{n+k}=$ $d x_{1} \wedge \ldots \wedge d x_{n+k}$ and $\sigma_{k}=d z_{1} \wedge \ldots \wedge d z_{k}$ where $x_{1}, \ldots, x_{n+k}$ and $z_{1}, \ldots$, $z_{k}$ are Cartesian coordinates in $\mathbb{C}^{n+k}$ and in $\mathbb{C}^{k}$ respectively. There exists (at least locally) an $n$-form $\sigma$ on $\mathbb{C}^{n}$ such that $\sigma_{n+k}=f^{*} \sigma_{k} \wedge \sigma$. Let $\varepsilon \notin \Sigma$. The restriction of the form $\sigma$ to the manifold $f_{\varepsilon}^{-1}(0)$ is well defined and is a volume form on $f_{\varepsilon}^{-1}(0)$. We also denote it by $\sigma$. Let $P_{1}, \ldots, P_{\nu}$ be the (non-degenerate) singular points of the 1 -form $\omega_{\varepsilon}$ on the $n$-dimensional manifold $f_{\varepsilon}^{-1}(0)$. For $\varepsilon \notin \Sigma$ and a germ $\varphi \in \mathcal{O}_{\mathbb{C}^{n}, 0}$, let

$$
R(\varphi, \varepsilon):=\sum_{i=1}^{\nu} \frac{\varphi\left(P_{i}\right)}{\widetilde{J}_{\varepsilon}\left(P_{i}\right)} .
$$

For a fixed $\varphi$ the function $R_{\varphi}(\varepsilon):=R(\varphi, \varepsilon)$ is holomorphic in the complement of the bifurcation diagram $\Sigma$.

In EG05c, it is proved that the function $R_{\varphi}(\varepsilon)$ has removable singularities on the bifurcation diagram $\Sigma$. This means that $R_{\varphi}(\varepsilon)$ can be extended to a holomorphic function on $\left(\mathbb{C}_{\varepsilon}^{M}, 0\right)$ (which we denote by the same symbol). Let

$$
R(\varphi):=R_{\varphi}(0) .
$$

This defines a linear function $R$ on $\mathcal{O}_{\mathbb{C}^{n}, 0}$. One can show that this linear function vanishes on the ideal $I$ and hence defines a linear function $R$ on the algebra $\mathcal{A}$. Using this function, we can define a quadratic form

$$
Q(\varphi, \psi)=R(\varphi \cdot \psi)
$$

on the algebra $\mathcal{A}$ generalizing the Eisenbud-Levine-Khimshiashvili quadratic form defined for a smooth $V$.

A similar form can be defined on a module of differential forms associated to the pair $(V, \omega)$, see [EG05c]. A special case of such a quadratic form (for curve singularities) was considered in [MvS90]. In the smooth case this quadratic form can be identified with the one on the algebra $\mathcal{A}$ by an isomorphism of the underlying spaces. This is no longer true for an ICIS. In general, these two quadratic forms have even different ranks.

Let $g:\left(\mathbb{C}^{n+1}, 0\right) \rightarrow(\mathbb{C}, 0)$ be a real analytic function (i.e. a function taking real values on $\left.\mathbb{R}^{n+1} \subset \mathbb{C}^{n+1}\right)$ with an isolated singular point at 0 . Then there is the following topological formula for the index of the gradient vector field $\operatorname{grad} g$ at the origin. Let $\eta$ be a sufficiently small positive real number. The complex conjugation induces involutions on the Milnor fibres 
$M_{g, \eta}$ and $M_{g,-\eta}$ of $g$. Their actions on the homology groups $H_{n}\left(M_{g, \eta}\right)$ and $H_{n}\left(M_{g,-\eta}\right)$ will be denoted by $\sigma_{+}$and $\sigma_{-}$respectively. On these homology groups we have the intersection forms $\langle\cdot, \cdot\rangle$. We consider the symmetric bilinear forms $Q_{+}(a, b):=\left\langle\sigma_{+} a, b\right\rangle$ and $Q_{-}(a, b):=\left\langle\sigma_{-} a, b\right\rangle$ on $H_{n}\left(M_{g, \eta}\right)$ and $H_{n}\left(M_{g,-\eta}\right)$ respectively. The following statement was conjectured by Arnold Ar78, and proved in Gu84, Va85.

Theorem 12. If $n$ is even then

$$
\operatorname{ind}_{0} \operatorname{grad} g=(-1)^{\frac{n}{2}} \frac{1}{2}\left(\operatorname{sgn} Q_{-}-\operatorname{sgn} Q_{+}\right),
$$

where $\operatorname{grad} g$ is the gradient vector field of the function $g$ on $\mathbb{R}^{n+1}$.

If Var : $H_{n}\left(M_{g, \eta}, \partial M_{g, \eta}\right) \rightarrow H_{n}\left(M_{g, \eta}\right)$ denotes the variation operator of the singularity of $g$, then there is another formula for the index in [Gu84]:

Theorem 13. One has

$$
\text { ind }_{0} \operatorname{grad} g=(-1)^{\frac{n(n-1)}{2}} \operatorname{sgn} \operatorname{Var}^{-1} \sigma_{+} .
$$

In [EG99] a generalization of such a formula for the radial index of a gradient vector field on an algebraically isolated real analytic isolated complete intersection singularity in $\mathbb{C}^{n+k}$ was obtained.

In Se95, SS96] formulas are given evaluating the GSV index of a singular point of a vector field on an isolated hypersurface or complete intersection singularity on a resolution of the singularity.

A. Esterov has extended the method of A.Khovanski of computation of intersection numbers from complete intersections to determinantal varieties. Using this he gave formulae for the GSV index of a 1-form on an ICIS and for its generalization to collections of 1 -forms (see Section 8 ) in terms of Newton diagrams of the equations of the ICIS and of the components of the 1-forms. See Es05b] for a short account of the results (a full version has been submitted to a journal; see also Es05a for some preliminary results).

In K102 a residue formula for the GSV index of a holomorphic 1-form on an isolated surface singularity in the spirit of Theorem 10 is proved.

\section{INDICES OF MEROMORPHIC 1-FORMS}

Nonzero holomorphic vector fields (as well as holomorphic 1-forms) on a compact complex manifold rarely exist. Therefore it is interesting to consider meromorphic vector fields or 1-forms of which there are a lot (at least for projective manifolds). For a meromorphic vector field defined by a holomorphic section of the vector bundle $T M \otimes L$ ( $L$ is a holomorphic line bundle on $M$ ), the sum of the indices of its zeros is equal to the corresponding characteristic number $\left\langle c_{n}(T M \otimes L),[M]\right\rangle$ of the vector bundle $T M \otimes L$ (see, e.g., [BB70 ).

Let $\alpha$ be a meromorphic 1-form on a compact complex manifold $M^{n}$ which means that $\alpha$ is a holomorphic 1-form outside of a positive divisor $D$ and in a neighbourhood of each point of $M$ the form $\alpha$ can be written as $\widehat{\alpha} / F$ 
where $F=0$ is a local equation of the divisor $D$ and $\widehat{\alpha}$ is a holomorphic 1-form. Let $L$ be the line bundle associated to the divisor $D$, i.e., $L$ has a holomorphic section $s$ with zeros on $D$. Then $\omega=s \alpha$ is a holomorphic section of the vector bundle $T^{*} M \otimes L$. In some constructions (say, as in [BB70] ) one defines a meromorphic 1-form on $M$ simply as a holomorphic section of the tensor product $T^{*} M \otimes L$ for a holomorphic line bundle $L$. This definition is somewhat different from the one formulated above. E.g., in this case only the class of the divisor of poles of a meromorphic 1-form is defined, not the divisor itself. Moreover, in this setting, the value of a meromorphic 1-form on a vector field is not a function, but a section of the line bundle $L$. In the sequel we use the notation $\omega$ for a section of the vector bundle $T^{*} M \otimes L$, for short calling it a meromorphic 1-form as well. Suppose that the section $\omega$ has isolated zeros. Then the sum of their indices is equal to the characteristic number $\left\langle c_{n}\left(T^{*} M \otimes L\right),[M]\right\rangle$ and thus depends on $L$.

For a meromorphic 1-form on a smooth compact complex curve $M$ the characteristic number $\left\langle c_{1}\left(T^{*} M\right),[M]\right\rangle=-\chi(M)$ is equal to the number of zeros minus the number of poles counted with multiplicities. Therefore to express the Euler characteristic of a compact manifold in terms of singularities of a meromorphic 1-form one has to take the divisor $D$ of its poles into account as well. If a meromorphic 1-form on a manifold $M^{n}$ is defined simply as a section of $T^{*} M \otimes L$, the divisor of poles is not defined and thus one also cannot define singular points of the 1-form on its pole locus.

One would like to give a Poincaré-Hopf type formula for meromorphic 1-forms, i.e., to express the Euler characteristic of a compact manifold or of a smoothing of a complete intersection in terms of singularities of a meromorphic 1-form. For that it is possible to introduce a suitable notion of an index of a germ of a meromorphic 1-form (with an additional structure) on an ICIS, so that the indices of the singular points sum up to (plus-minus) the Euler characteristic of a smoothing. There are several descriptions of the index of a meromorphic 1-form, one of them being the alternating sum of the dimensions of certain algebras.

A Poincaré-Hopf type formula can be considered as one describing a localization of an invariant (say, of the Euler characteristic) of a manifold at singular points of, say, a vector field or a 1-form, i.e., a representation of the invariant as the sum of integer invariants ("indices") corresponding to singular points. Therefore one first has to define singular points. Let $M^{n}$ be a complex manifold, let $L$ be a line bundle on $M$, and let $\omega$ be a holomorphic section of the bundle $T^{*} M \otimes L$. One has zeros of $\omega$ on $M$, but the pole locus of $\omega$ is not well defined. In order to discuss singular points of $\omega$ on its pole locus (which is necessary as we saw in the example when $M$ was a curve), we have to fix this locus. This means that we have to choose a holomorphic section $s=s_{1}$ of the line bundle $L$ or to choose its zero divisor $D=D_{1}$. One can say that we have to consider $\alpha=\omega / s$, i.e., to proceed with our initial definition of a meromorphic 1-form. 
For the further setting we suppose that the divisor $D$ of poles of the 1form $\omega$ is non-singular (in particular, reduced). This is not very essential for this section, however, this makes the discussion simpler. Since $D$ is a submanifold of $M$, there is a well defined map $T^{*} M \mid D \rightarrow T^{*} D$ and thus $\left.\left(T^{*} M \otimes L\right)\right|_{D} \rightarrow T^{*} D \otimes L{ }_{D}$ (the restriction of meromorphic 1-forms to $D$ ). (Using the 1-form $\alpha$, one can also define only a section of the vector bundle $T^{*} D \otimes L{ }_{D}$, but not a meromorphic 1-form on $D$ with precisely defined pole locus.)

Let $\omega_{1}$ be the restriction of $\omega$ to $D_{1}$. It is a holomorphic section of the vector bundle $T^{*} D \otimes L{ }_{D}$. Its zeros are well defined and should be considered as singular points of the meromorphic 1-form $\omega$ on the pole locus. To discuss its singular points on its pole locus we again have to fix a divisor. Suppose that there exists a (positive) divisor $D_{2}$ on $M$ which is the zero locus of another section $s_{2}$ of the same line bundle $L$ and which intersects $D_{1}$ transversally (in particular, this means that $D_{2}$ is non-singular at its intersection points with $D_{1}$ and $D_{1} \cap D_{2}$ is non-singular as well). One has the restriction of $\omega$ to $D_{1} \cap D_{2}$ and its zeros there.

Going on this way we arrive at the situation when we have fixed $n$ divisors $D_{1}, \ldots, D_{n}$ (zeros of sections $s_{1}, \ldots, s_{n}$ of the line bundle $L$ ) so that, for each $i=1, \ldots n, D_{1} \cap \ldots \cap D_{i}$ is non-singular. The set of singular points of the 1 -form $\omega$ is the union of the zeros of $\omega$ itself and of the restrictions of $\omega$ to $D_{1} \cap \ldots \cap D_{i}$ for all $i=1, \ldots, n$. (For $i=n$ the intersection $D_{1} \cap \ldots \cap D_{n}$ is zero-dimensional and all its points should be considered as zeros of the 1 -form $\omega$.) One can say that we have to consider a collection of meromorphic 1 -forms $\omega / s_{1}, \ldots, \omega / s_{n}$ on $M$ proportional to each other.

There is the following Poincaré-Hopf type formula for meromorphic 1forms. Let $M^{n}$ be a compact complex manifold, let $\omega$ be a meromorphic 1-form on $M$, that is, a holomorphic section of the bundle $T^{*} M \otimes L$ where $L$ is a holomorphic line bundle with nonzero holomorphic sections. Suppose that $D_{1}=D, D_{2}, \ldots, D_{n}$ are zero divisors of holomorphic sections of the line bundle $L$ such that, for each $i=1, \ldots, n, D_{1} \cap \ldots \cap D_{i}$ is non-singular. Suppose that the form $\omega$ itself and its restrictions to the submanifolds $D_{1} \cap$ $\ldots \cap D_{i}, i=1, \ldots, n$, have only isolated zeros. Let $m_{0}$ (respectively $m_{i}$, $i=1, \ldots, n)$ be the number of zeros of the form $\omega$ (respectively, of the restriction of $\omega$ to the intersection $D_{1} \cap \ldots \cap D_{i}$ ) counted with multiplicities. In particular $m_{n}$ is the number of points in $D_{1} \cap \ldots \cap D_{n}$.

Theorem 14. $c_{n}\left(T^{*} M\right)[M]=(-1)^{n} \chi\left(M^{n}\right)=m_{0}-m_{1}+\ldots+(-1)^{n} m_{n}$.

Remark. One can show that

$$
m_{0}=(-1)^{n}(\chi(M)-\chi(D))=(-1)^{n} \chi(M \backslash D) .
$$

Suppose that all zeros of $\omega$ are outside of $D$ (one can consider this situation as the generic one). Let $s$ be the holomorphic section of the line bundle $L$ with zeros on $D$. Then $\alpha=\omega / s$ is a holomorphic 1 -form on $M \backslash D$ with simple poles along $D$. So in this case the number $m_{0}$ of zeros of the 
holomorphic 1-form $\alpha$ on $M \backslash D$ coincides with $(-1)^{n} \chi(M \backslash D)$. This is the relation which holds for holomorphic 1-forms on compact manifolds ( $M \backslash D$ is not compact).

Example 4. Let

$$
\alpha=\frac{x d y-y d x+d z}{x^{2}+4 y^{2}+z^{2}+1}
$$

be a meromorphic 1 -form on the projective space $\mathbb{C P}^{3}(x, y, z$ are affine coordinates). One can see that the zeros of the corresponding $\omega$ on $\mathbb{C P}^{3}$ and also the zeros of $\left.\omega\right|_{D}, D=D_{1}=\left\{x^{2}+4 y^{2}+z^{2}+1=0\right\}$, are isolated and $m_{0}=0, m_{1}=4$. To define other singular points one has to choose $D_{2}$ and $D_{3}$ (e.g., $D_{2}=\left\{x^{2}+y^{2}+4 z^{2}=0\right\}, D_{3}=\left\{x^{2}+y^{2}+z^{2}=0\right\}$ ). One has $m_{2}=8, m_{3}=8,0-4+8-8=-4=(-1)^{3} \chi\left(\mathbb{C P}^{3}\right)$. Note that as a meromorphic 1 -form on $\mathbb{C P}^{3}$ with poles on the hypersurface $P_{d}(x, y, z)=0$, $\operatorname{deg} P_{d}=d$, it is natural to take

$$
\frac{A_{d-2} d x+B_{d-2} d y+C_{d-2} d z}{P_{d}},
$$

where $A_{d-2}, B_{d-2}$, and $C_{d-2}$ are polynomials in $x, y, z$ of degree $d-2$ ( $\alpha$ is not of this form). However, one can show that such a 1-form has non-isolated zeros on $\mathbb{C P}^{3}$ (at infinity).

Now let $V^{n}$ be a compact subvariety of a complex manifold $M^{n+k}$ such that in a neighbourhood of each point $V$ is a complete intersection in $M$ with only isolated singularities ( $M$ is not supposed to be compact). Let $\omega$ be a meromorphic 1-form on $M$, i.e., a holomorphic section of the bundle $T^{*} M \otimes L$, where $L$ is a line bundle on $M$ with nonzero holomorphic sections. By a smoothing $\widetilde{V}$ of $V$ we understand a smooth $\left(C^{\infty}\right)$ manifold which is obtained from $V$ by smoothing its singular points (which are ICIS) in the usual way (as complex ICIS). (If $V$ is a complete intersection in the projective space $\mathbb{C P}^{n+k}$, a smoothing of $V$ can be obtained as an analytic submanifold of $\mathbb{C P}^{n+k}$ as well.)

It is possible to formulate a Poincaré-Hopf type formula for the Euler characteristic of $V$ and of its smoothing $\widetilde{V}$ in terms of singular points of the 1-form $\omega$ on $V$. As above we suppose that there exist $n$ holomorphic sections $s_{1}, \ldots, s_{n}$ of the line bundle $L$ with zero divisors $D_{1}, \ldots, D_{n}$ such that, for each $i=1, \ldots, n$, the intersection $V \cap D_{1} \cap \ldots \cap D_{i}$ is of dimension $n-i$ and has only isolated singularities (and thus is reduced for $i<n)$. We also suppose that the restriction of $\omega$ to the non-singular part of $V$ and its restriction to the non-singular part of $V \cap D_{1} \cap \ldots \cap D_{i}$ $(i=1, \ldots, n)$ have only isolated zeros. As singular points of the 1 -form $\omega$ on $V$ we consider all zeros of its restrictions to the non-singular parts of $V$ and of the intersections $V \cap D_{1} \cap \ldots \cap D_{i}(i=1, \ldots, n)$ and all singular points of $V$ and of $V \cap D_{1} \cap \ldots \cap D_{i}$ as well.

Let $P$ be a singular point of the 1 -form $\omega$ on $V$. Suppose that the divisors $D_{1}, \ldots, D_{\ell}(0 \leq \ell \leq n)$ pass through the point $P$ and (if $\left.\ell<n\right) D_{\ell+1}$ does 
not. In a neighbourhood of the point $P$, in some local coordinates on $M$ centred at the point $P$, we have the following situation. The variety (ICIS) $V$ (possibly non-singular) is defined by $k$ equations $f_{1}=\ldots=f_{k}=0$. Let divisors $D_{1}, \ldots, D_{\ell}$ be defined by equations $f_{k+1}=0, \ldots, f_{k+\ell}=0$. Let us remind that $\left\{f_{1}=\ldots=f_{k}=f_{k+1}=\ldots=f_{k+i}=0\right\}$ is an ICIS for each $i=0,1, \ldots, \ell$. After choosing a local trivialization of the line bundle $L$, the 1-form $\omega$ can be written as $\sum_{i=1}^{n+k} A_{i}(x) d x_{i}$ where $A_{i}(x)$ are holomorphic. One can say that we consider a collection of meromorphic 1-forms $\omega / f_{k+1}$, $\ldots, \omega / f_{k+\ell}$ on $V$ proportional to each other. Let us denote the set of local data $\left(V, \omega, f_{k+1}, \ldots, f_{k+\ell}\right)$ by $\Omega$.

Let ind ${ }^{(0)} \Omega$ (respectively ind ${ }^{(i)} \Omega, i=1, \ldots, \ell$ ) be the index of the holomorphic 1-form $\omega$ on $V$ (respectively on the ICIS $V \cap D_{1} \cap \ldots \cap D_{i}$ ) defined in Section 3 ,

Definition: The alternating sum

$$
\operatorname{ind}_{P} \Omega=\operatorname{ind}^{(0)} \Omega-\text { ind }^{(1)} \Omega+\ldots+(-1)^{\ell} \operatorname{ind}^{(\ell)} \Omega
$$

is called the index of the 1 -form $\omega$ at the point $P$ with respect to the divisors $D_{1}, \ldots, D_{n}$.

According to the statements from Section 3 (see also Section 6] for the last point) one has the following three equivalent descriptions of the index $\operatorname{ind}_{P} \Omega$.

1. Let $B_{\delta}$ be the ball of sufficiently small radius $\delta$ centred at the origin in $\mathbb{C}^{n+k}$ and let $S_{\delta}$ be its boundary. Let $\varepsilon=\left(\varepsilon_{1}, \ldots, \varepsilon_{k}, \ldots, \varepsilon_{k+\ell}\right) \in\left(\mathbb{C}^{k+\ell}, 0\right)$ be small enough and such that $\varepsilon^{(k+i)}=\left(\varepsilon_{1}, \ldots, \varepsilon_{k}, \ldots, \varepsilon_{k+i}\right)$ is not a critical value of the map $F_{k+i}=\left(f_{1}, \ldots, f_{k}, \ldots, f_{k+i}\right):\left(\mathbb{C}^{n+k}, 0\right) \rightarrow\left(\mathbb{C}^{k+i}, 0\right)$ for each $i=0,1, \ldots, \ell$. The restriction of the 1 -form $\omega$ to the smooth manifold $V_{i}=F_{k+i}^{-1}\left(\varepsilon^{(k+i)}\right)$ has isolated zeros in $B_{\delta}$. Let $m_{i}$ be the number of them counted with multiplicities. Then ind ${ }^{(i)} \Omega=m_{i}$, ind $\operatorname{in}_{P}=m_{0}-m_{1}+\ldots+$ $(-1)^{\ell} m_{\ell}$.

2. Let $K_{i}(i=0,1, \ldots, \ell)$ be the link of the ICIS $V \cap D_{1} \cap \ldots \cap D_{i}$, i.e., the intersection $V \cap D_{1} \cap \ldots \cap D_{i} \cap S_{\delta}$. Let $d_{i}$ be the degree of the map

$$
\left(\omega, d f_{1}, \ldots, d f_{k}, \ldots, d f_{k+i}\right): K_{i} \rightarrow W_{k+i+1}\left(\mathbb{C}^{n+k}\right)
$$

$\left(W_{k+i+1}\left(\mathbb{C}^{n+k}\right)\right.$ is the Stiefel manifold of $(k+i+1)$-frames in the dual $\left.\mathbb{C}^{n+k}\right)$. Then ind ${ }^{(i)} \Omega=d_{i}, \operatorname{ind}_{P} \Omega=d_{0}-d_{1}+\ldots+(-1)^{\ell} d_{\ell}$.

3. Let $I_{i}(i=0,1, \ldots, \ell)$ be the ideal of the ring $\mathcal{O}_{\mathbb{C}^{n+k}, 0}$ of germs of holomorphic functions of $n+k$ variables at the origin generated by $f_{1}, \ldots$, $f_{k}, \ldots, f_{k+i}$ and the $(k+i+1) \times(k+i+1)$-minors of the matrix

$$
\left(\begin{array}{ccc}
\frac{\partial f_{1}}{\partial x_{1}} & \cdots & \frac{\partial f_{1}}{\partial x_{n+k}} \\
\vdots & \ddots & \vdots \\
\frac{\partial f_{k+i}}{\partial x_{1}} & \cdots & \frac{\partial f_{k+i}}{\partial x_{n+k}} \\
A_{1} & \cdots & A_{n+k}
\end{array}\right) .
$$


Let $\nu_{i}=\operatorname{dim}_{\mathbb{C}} \mathcal{O}_{\mathbb{C}^{n+k}, 0} / I_{i}$. Then ind ${ }^{(i)} \Omega=\nu_{i}$, ind ${ }_{P} \omega=\nu_{0}-\nu_{1}+\ldots+(-1)^{\ell} \nu_{\ell}$.

One has the following Poincaré-Hopf type formula.

Theorem 15.

$$
\sum_{P} \operatorname{ind}_{P} \Omega=(-1)^{n} \chi(\widetilde{V})
$$

where $\widetilde{V}$ is a smoothing of the variety $V$.

In order to get the Euler characteristic of the variety $V$ itself one has to correct this formula by taking the Milnor numbers of the singular points of $V$ into account. For $P \in V$, let $\mu_{P}$ denote the Milnor number of the ICIS $V$ at the point $P$ (see, e.g., Lo84). Note that, if $V$ is non-singular at $P$, then $\mu_{P}=0$.

\section{Theorem 16.}

$$
(-1)^{n} \chi(V)=\sum_{P}\left(\operatorname{ind}_{P} \Omega-\mu_{P}\right)
$$

Remark. The Milnor number $\mu_{P}$ can also be written as an alternating sum of indices of holomorphic 1-forms on several ICIS. One can say that the term $\mu_{P}$ corresponds to the difference between two possible definitions of the index as in [SS98, Proposition 1.4].

In some cases it is natural to consider the situation when the pole locus of the meromorphic 1-form $\omega$ is a multiple of an irreducible divisor. Examples are:

1) A polynomial 1-form on $\mathbb{C}^{n}$ is a meromorphic 1 -form on $\mathbb{C} \mathbb{P}^{n}$ the pole locus of which is a multiple of the infinite hyperplane $\mathbb{C P}_{\infty}^{n-1}$.

2) Let $f$ be a meromorphic function with the pole locus $k D$ where $D$ is an irreducible divisor (i.e., in a neighbourhood of each point $f$ can be written as $\tilde{f} / F^{k}$ where $\tilde{f}$ is holomorphic and $F=0$ is a local equation of $D)$. Then its differential $d f$ is a meromorphic 1 -form with the pole locus $(k+1) D$ (i.e., in a neighbourhood of each point $d f$ can be written as $\widetilde{\omega} / F^{k+1}$ where $\widetilde{\omega}$ is a holomorphic 1-form). A polynomial function of degree $k$ on $\mathbb{C}^{n}$ is a meromorphic function on $\mathbb{C P}^{n}$ with the pole locus $k \mathbb{C P}_{\infty}^{n-1}$.

Let us give a version of Theorem 14 for this case. The fact that the pole divisor of a 1-form is multiple means that the corresponding line bundle is a power of another one. Let $\omega$ be a meromorphic 1-form on a compact complex manifold $M^{n}$, that is, a holomorphic section of the bundle $T^{*} M \otimes L$, where $L=\lambda^{k}, k>1$. Suppose that $D_{1}=D, D_{2}, \ldots, D_{n}$ are zero divisors of holomorphic sections of the line bundle $\lambda$ such that, for each $i=1, \ldots, n$, $D_{1} \cap \ldots \cap D_{i}$ is non-singular and the form $\omega$ itself and its restrictions to $D_{1} \cap \ldots \cap D_{i}$ have isolated zeros. Let $m_{0}$ (respectively $m_{i}, i=1, \ldots, n$ ) be the number of zeros of the 1 -form $\omega$ (respectively of its restriction to $\left.D_{1} \cap \ldots \cap D_{i}\right)$ counted with multiplicities. 


\section{Theorem 17.}

$$
\left\langle c_{n}\left(T^{*} M\right),[M]\right\rangle=(-1)^{n} \chi(M)=m_{0}-k m_{1}+\ldots+(-1)^{n} k m_{n} .
$$

Remark. In HS98, Theorem 3.1] there is given a formula for the sum of the residues corresponding to the singular points of the foliation on the projective plane $\mathbb{C P}^{2}$ given by $d f=0$, where $f$ is a polynomial function of degree $k$ on $\mathbb{C}^{2}$ (which defines a meromorphic function on $\mathbb{C P}^{2}$ ). In our terms this is the formula for the number $m_{0}$ of zeros of the meromorphic 1 -form $d f$, the pole locus of which is $k+1$ times the infinite line. Thus Theorem 17 can be considered as a generalization of [HS98, Theorem 3.1] to higher dimensions and to meromorphic 1-forms which are not, in general, differentials of functions.

\section{INDICES OF COLLECTIONS OF 1-FORMS}

One can say that all the indices discussed above are connected with the Euler characteristic (e.g. through the Poincaré-Hopf type theorem). If $M$ is a complex analytic manifold of dimension $n$, then its Euler characteristic $\chi(M)$ is the characteristic number $\left\langle c_{n}(T M),[M]\right\rangle=(-1)^{n}\left\langle c_{n}\left(T^{*} M\right),[M]\right\rangle$, where $T M$ is the tangent bundle of the manifold $M, T^{*} M$ is the dual bundle, and $c_{n}$ is the corresponding Chern class. One can try to find generalizations of some notions and statements about indices of vector fields or of 1-forms for other characteristic numbers (different from $\left\langle c_{n}(T M),[M]\right\rangle$ or $\left.\left\langle c_{n}\left(T^{*} M\right),[M]\right\rangle\right)$. This was made in EG05b and EG05d for the GSV index and for the Euler obstruction.

The top Chern class of a vector bundle is the (first) obstruction to existence of a non-vanishing section. Other Chern classes are obstructions to existence of linear independent collections of sections. Therefore instead of 1 -forms on a complex variety we consider collections of 1-forms.

Let $\pi: E \rightarrow M$ be a complex analytic vector bundle of rank $m$ over a complex analytic manifold $M$ of dimension $n$. It is known that the $(2(n-$ $k)$-dimensional) cycle Poincaré dual to the characteristic class $c_{k}(E)(k=$ $1, \ldots, m)$ is represented by the set of points of the manifold $M$ where $m-k+1$ generic sections of the vector bundle $E$ are linearly dependent (cf., e.g., GH78, p. 413]).

Let $\mathcal{M}(p, q), D_{p, q}$, and $W_{p, q}$ be the spaces defined in Section 3. Let $\mathbf{k}=$ $\left(k_{1}, \ldots, k_{s}\right)$ be a sequence of positive integers with $\sum_{i=1}^{s} k_{i}=k$. Consider the space $\mathcal{M}_{m, \mathbf{k}}=\prod_{i=1}^{s} \mathcal{M}\left(m, m-k_{i}+1\right)$ and the subvariety $D_{m, \mathbf{k}}=$ $\prod_{i=1}^{s} D_{m, m-k_{i}+1}$ in it. The variety $D_{m, \mathbf{k}}$ consists of sets $\left\{A_{i}\right\}$ of $m \times(m-$ $\left.k_{i}+1\right)$ matrices such that $\operatorname{rk} A_{i}<m-k_{i}+1$ for each $i=1, \ldots, s$. Since $D_{m, \mathbf{k}}$ is irreducible of codimension $k$, its complement $W_{m, \mathbf{k}}=\mathcal{M}_{m, \mathbf{k}} \backslash D_{m, \mathbf{k}}$ is $(2 k-2)$-connected, $H_{2 k-1}\left(W_{m, \mathbf{k}}\right) \cong \mathbb{Z}$, and there is a natural choice of a generator of the latter group. This choice defines a degree (an integer) of a map from an oriented manifold of dimension $2 k-1$ to the manifold $W_{m, \mathbf{k}}$. 
Let $(V, 0) \subset\left(\mathbb{C}^{N}, 0\right)$ be an $n$-dimensional isolated complete intersection singularity (ICIS) defined by equations $f_{1}=\ldots=f_{N-n}=0\left(f_{i} \in \mathcal{O}_{\mathbb{C}^{N}, 0}\right)$. Let $f$ be the analytic map $\left(f_{1}, \ldots, f_{N-n}\right):\left(\mathbb{C}^{N}, 0\right) \rightarrow\left(\mathbb{C}^{N-n}, 0\right)(V=$ $\left.f^{-1}(0)\right)$. Let $\left\{X_{j}^{(i)}\right\}$ be a collection of vector fields on a neighbourhood of the origin in $\left(\mathbb{C}^{N}, 0\right)\left(i=1, \ldots, s ; j=1, \ldots, n-k_{i}+1 ; \sum k_{i}=n\right)$ which are tangent to the ICIS $(V, 0)=\left\{f_{1}=\cdots=f_{N-n}=0\right\} \subset\left(\mathbb{C}^{N}, 0\right)$ at non-singular points of $V$. We say that a point $p \in V \backslash\{0\}$ is non-singular for the collection $\left\{X_{j}^{(i)}\right\}$ on $V$ if at least for some $i$ the vectors $X_{1}^{(i)}(p), \ldots, X_{n-k_{i}+1}^{(i)}(p)$ are linearly independent. Suppose that the collection $\left\{X_{j}^{(i)}\right\}$ has no singular points on $V$ outside of the origin in a neighbourhood of it. Let $U$ be a neighbourhood of the origin in $\mathbb{C}^{N}$ where all the functions $f_{r}(r=1, \ldots, N-n)$ and the vector fields $X_{j}^{(i)}$ are defined and such that the collection $\left\{X_{j}^{(i)}\right\}$ has no singular points on $(V \cap U) \backslash\{0\}$. Let $S_{\delta} \subset U$ be a sufficiently small sphere around the origin which intersects $V$ transversally and denote by $K=V \cap S_{\delta}$ the link of the ICIS $(V, 0)$. The manifold $K$ has a natural orientation as the boundary of a complex analytic manifold. Let $\Psi_{V}$ be the mapping from $V \cap U$ to $\mathcal{M}_{n, \mathbf{k}}$ which sends a point $x \in V \cap U$ to the collection of $N \times\left(N-k_{i}+1\right)$-matrices

$$
\left\{\left(\operatorname{grad} f_{1}(x), \ldots, \operatorname{grad} f_{N-n}(x), X_{1}^{(i)}(x), \ldots, X_{n-k_{i}+1}^{(i)}(x)\right)\right\}, \quad i=1, \ldots, s .
$$

Here $\operatorname{grad} f_{r}$ is the gradient vector field of $f_{r}$ defined in Section 3 . Its restriction $\psi_{V}$ to the link $K$ maps $K$ to the subset $W_{N, \mathbf{k}}$.

Definition: The index $\operatorname{ind}_{V, 0}\left\{X_{j}^{(i)}\right\}$ of the collection of vector fields $\left\{\omega_{j}^{(i)}\right\}$ on the ICIS $V$ is the degree of the mapping $\psi_{V}: K \rightarrow W_{N, \mathbf{k}}$.

For $s=1, k_{1}=n$, this index is the GSV index of a vector field on an ICIS (see Section B).

Let $V \subset \mathbb{C P}^{N}$ be an $n$-dimensional complete intersection with isolated singular points, $V=\left\{f_{1}=\ldots=f_{N-n}=0\right\}$ where $f_{i}$ are homogeneous functions in $(N+1)$ variables. Let $\left\{X_{j}^{(i)}\right\}$ be a collection of continuous vector fields on $\mathbb{C P}^{N}$ which are tangent to $V$. Let $\widetilde{V}$ be a smoothing of the complete intersection $V$, i.e. $\widetilde{V}$ is defined by $N-n$ equations $\widetilde{f}_{1}=\ldots=\widetilde{f}_{N-n}=0$ where the homogeneous functions $\widetilde{f}_{i}$ are small perturbations of the functions $f_{i}$ and $\widetilde{V}$ is smooth.

The usual description of Chern classes of a vector bundle as obstructions to existence of several linear independent sections of the bundle implies the following statement.

Theorem 18. One has

$$
\sum_{p \in V} \operatorname{ind}_{p}\left\{X_{j}^{(i)}\right\}=\left\langle\prod_{i=1}^{s} c_{k_{i}}(T \widetilde{V}),[\tilde{V}]\right\rangle,
$$

where $\widetilde{V}$ is a smoothing of the complete intersection $V$. 
Now let $\left\{\omega_{j}^{(i)}\right\}$ be a collection of (continuous) 1-forms on a neighbourhood of the origin in $\left(\mathbb{C}^{N}, 0\right)$ with $i=1, \ldots, s, j=1, \ldots, n-k_{i}+1, \sum k_{i}=n$. We say that a point $p \in V \backslash\{0\}$ is non-singular for the collection $\left\{\omega_{j}^{(i)}\right\}$ on $V$ if at least for some $i$ the restrictions of the 1-forms $\omega_{j}^{(i)}(p)$ to the tangent space $T_{p} V$ are linearly independent. Suppose that the collection $\left\{\omega_{j}^{(i)}\right\}$ has no singular points on $V$ outside of the origin in a neighbourhood of it. As above let $K=V \cap S_{\delta}$ be the link of the ICIS $(V, 0)$ (all the functions $f_{r}$ and the 1-forms $\omega_{j}^{(i)}$ are defined in a neighbourhood of the ball $B_{\delta}$. Let $\Psi_{V}$ be the mapping from $V \cap U$ to $\mathcal{M}_{n, \mathbf{k}}$ which sends a point $x \in V \cap U$ to the collection of $N \times\left(N-k_{i}+1\right)$-matrices

$$
\left\{\left(d f_{1}(x), \ldots, d f_{N-n}(x), \omega_{1}^{(i)}(x), \ldots, \omega_{n-k_{i}+1}^{(i)}(x)\right)\right\}, \quad i=1, \ldots, s .
$$

Its restriction $\psi_{V}$ to the link $K$ maps $K$ to the subset $W_{N, \mathbf{k}}$.

Definition: The index $\operatorname{ind}_{V, 0}\left\{\omega_{j}^{(i)}\right\}$ of the collection of 1 -forms $\left\{\omega_{j}^{(i)}\right\}$ on the ICIS $V$ is the degree of the mapping $\psi_{V}: K \rightarrow W_{N, \mathbf{k}}$.

One can easily see that the index $\operatorname{ind}_{V, 0}\left\{\omega_{j}^{(i)}\right\}$ is equal to the intersection number of the germ of the image of the mapping $\Psi_{V}$ with the variety $D_{N, \mathbf{k}}$. If all the 1 -forms $\omega_{j}^{(i)}$ are complex analytic, the mapping $\Psi_{V}$ is complex analytic as well.

For $s=1, k_{1}=n$, this index is the GSV index of a 1-form (Section 3).

Let $V \subset \mathbb{C P}^{N}$ be an $n$-dimensional complete intersection with isolated singular points, $V=\left\{f_{1}=\ldots=f_{N-n}=0\right\}$ where $f_{i}$ are homogeneous functions in $(N+1)$ variables. Let $L$ be a complex line bundle on $V$ and let $\left\{\omega_{j}^{(i)}\right\}$ be a collection of continuous 1-forms on $V$ with values in $L$. Here this means that the forms $\omega_{j}^{(i)}$ are continuous sections of the vector bundle $T^{*} V \otimes L$ outside of the singular points of $V$. Since, in a neighbourhood of each point $p$, the vector bundle $L$ is trivial, one can define the index $\operatorname{ind}_{p}\left\{\omega_{j}^{(i)}\right\}$ of the collection of 1-forms $\left\{\omega_{j}^{(i)}\right\}$ at the point $p$ just in the same way as in the local setting above. Let $\widetilde{V}$ be a smoothing of the complete intersection $V$. One can consider $L$ as a line bundle on the smoothing $\widetilde{V}$ of the complete intersection $V$ as well (e.g., using the pull back along a projection of $\widetilde{V}$ to $V ; L$ is not, in general, complex analytic). The collection $\left\{\omega_{j}^{(i)}\right\}$ of 1 -forms can also be extended to a neighbourhood of $V$ in such a way that it will define a collection of 1-forms on the smoothing $\widetilde{V}$ (also denoted by $\left\{\omega_{j}^{(i)}\right\}$ ) with isolated singular points. The sum of the indices of the collection $\left\{\omega_{j}^{(i)}\right\}$ on the smoothing $\widetilde{V}$ of $V$ in a neighbourhood of the point $p$ is equal to the index $\operatorname{ind}_{V, p}\left\{\omega_{j}^{(i)}\right\}$.

One has the following analogue of Proposition 4 for 1-forms. 
Theorem 19. One has

$$
\sum_{p \in V} \operatorname{ind}_{p}\left\{\omega_{j}^{(i)}\right\}=\left\langle\prod_{i=1}^{s} c_{k_{i}}\left(T^{*} \widetilde{V} \otimes L\right),[\widetilde{V}]\right\rangle,
$$

where $\widetilde{V}$ is a smoothing of the complete intersection $V$.

As above, let $(V, 0) \subset\left(\mathbb{C}^{N}, 0\right)$ be the ICIS defined by the equations $f_{1}=$ $\cdots=f_{N-n}=0$. Let $\left\{\omega_{j}^{(i)}\right\}\left(i=1, \ldots, s ; j=1, \ldots, n-k_{i}+1\right)$ be a collection of 1 -forms on a neighbourhood of the origin in $\mathbb{C}^{N}$ without singular points on $V \backslash\{0\}$ in a neighbourhood of the origin. If all the 1-forms $\omega_{j}^{(i)}$ are complex analytic, there exists an algebraic formula for the index $\operatorname{ind}_{V, 0}\left\{\omega_{j}^{(i)}\right\}$ of the collection $\left\{\omega_{j}^{(i)}\right\}$ similar to that from Theorem 11].

Let $I_{V,\left\{\omega_{j}^{(i)}\right\}}$ be the ideal in the ring $\mathcal{O}_{\mathbb{C}^{N}, 0}$ generated by the functions $f_{1}, \ldots, f_{N-n}$ and by the $\left(N-k_{i}+1\right) \times\left(N-k_{i}+1\right)$ minors of all the matrices

$$
\left(d f_{1}(x), \ldots, d f_{N-n}(x), \omega_{1}^{(i)}(x), \ldots, \omega_{n-k_{i}+1}^{(i)}(x)\right)
$$

for all $i=1, \ldots, s$.

Theorem 20. (see EG05b])

$$
\operatorname{ind}_{V, 0}\left\{\omega_{j}^{(i)}\right\}=\operatorname{dim}_{\mathbb{C}} \mathcal{O}_{\mathbb{C}^{N}, 0} / I_{V,\left\{\omega_{j}^{(i)}\right\}}
$$

Remark. A formula similar to that of Theorem 20 does not exist for collections of vector fields. A reason is that in this case the index is the intersection number with $D_{N, \mathbf{k}}$ of the image of the ICIS $(V, 0)$ under a map which is not complex analytic. Moreover, in some cases this index can be negative (see e.g. GSV91, Proposition 2.2]).

Example 5. Let $V$ be the (singular) quadric $x_{1}^{2}+x_{2}^{2}+x_{3}^{2}=0$ in the projective space $\mathbb{C P}^{3}$ with the coordinates $\left(x_{0}: x_{1}: x_{2}: x_{3}\right)$. Let the 1 forms from the collection $\left\{\left\{\omega_{1}^{(1)}, \omega_{2}^{(1)}\right\},\left\{\omega_{1}^{(2)}, \omega_{2}^{(2)}\right\}\right\}$ be defined in the affine chart $\mathbb{C}^{3}=\left\{x_{0}=1\right\}$ of the projective space $\mathbb{C P}^{3}$ as $\omega_{1}^{(1)}=d x_{1}, \omega_{2}^{(1)}=$ $x_{2} d x_{3}-x_{3} d x_{2}, \omega_{1}^{(2)}=d x_{2}, \omega_{2}^{(2)}=x_{1} d x_{3}-x_{3} d x_{1}$. They are holomorphic 1 -forms on the projective space $\mathbb{C P}^{3}$ with values in the line bundle $\mathcal{O}(2)$. It is easy to see that being restricted to the quadric $V$ the indicated collection of 1 -forms has no singular points outside of the singular point $(1: 0: 0: 0)$ of $V$ itself. Theorem [19] says that the index of this point is equal to $\left\langle c_{1}^{2}\left(T^{*} \widetilde{V} \otimes\right.\right.$ $\left.\left.i^{*} \mathcal{O}(2)\right),[\widetilde{V}]\right\rangle$, where $\widetilde{V}$ is a non-singular quadric, $i^{*} \mathcal{O}(2)$ is the restriction of the line bundle $\mathcal{O}(2)$ to $\widetilde{V}$. Taking into account that $\widetilde{V} \cong\left(\mathbb{C P}^{1}\right)^{2}$, one gets that this characteristic number is equal to 8 . Now by Theorem 20 the index of the indicated collection of 1-forms on the quadric $V$ at the origin in $\mathbb{C}^{3} \subset \mathbb{C P}^{3}$ is equal to $\operatorname{dim}_{\mathbb{C}} \mathcal{O}_{\mathbb{C}^{3}, 0} /\left\langle x_{1}^{2}+x_{2}^{2}+x_{3}^{2}, x_{2}^{2}+x_{3}^{2}, x_{1}^{2}+x_{3}^{2}\right\rangle$ which is also equal to 8 . 
There exists a generalization of the notion of the Euler obstruction to collections of 1-forms corresponding to different Chern numbers.

Let $\left(V^{n}, 0\right) \subset\left(\mathbb{C}^{N}, 0\right)$ be the germ of a purely $n$-dimensional reduced complex analytic variety at the origin (generally speaking with a non-isolated singularity). Let $\mathbf{k}=\left\{k_{i}\right\}, i=1, \ldots, s$, be a fixed partition of $n$ (i.e., $k_{i}$ are positive integers, $\left.\sum_{i=1}^{s} k_{i}=n\right)$. Let $\left\{\omega_{j}^{(i)}\right\}\left(i=1, \ldots, s, j=1, \ldots, n-k_{i}+1\right)$ be a collection of germs of 1 -forms on $\left(\mathbb{C}^{N}, 0\right.$ ) (not necessarily complex analytic; it suffices that the forms $\omega_{j}^{(i)}$ are complex linear functions on $\mathbb{C}^{N}$ continuously depending on a point of $\left.\mathbb{C}^{N}\right)$. Let $\varepsilon>0$ be small enough so that there is a representative $V$ of the germ $(V, 0)$ and representatives $\omega_{j}^{(i)}$ of the germs of 1 -forms inside the ball $B_{\varepsilon}(0) \subset \mathbb{C}^{N}$.

Definition: A point $P \in V$ is called a special point of the collection $\left\{\omega_{j}^{(i)}\right\}$ of 1-forms on the variety $V$ if there exists a sequence $\left\{P_{m}\right\}$ of points from the non-singular part $V_{\text {reg }}$ of the variety $V$ such that the sequence $T_{P_{m}} V_{\text {reg }}$ of the tangent spaces at the points $P_{m}$ has a limit $L$ (in $G(n, N)$ ) and the restrictions of the 1 -forms $\omega_{1}^{(i)}, \ldots, \omega_{n-k_{i}+1}^{(i)}$ to the subspace $L \subset T_{P} \mathbb{C}^{N}$ are linearly dependent for each $i=1, \ldots, s$. The collection $\left\{\omega_{j}^{(i)}\right\}$ of 1 -forms has an isolated special point on $(V, 0)$ if it has no special points on $V$ in a punctured neighbourhood of the origin.

Remark. If the 1 -forms $\omega_{j}^{(i)}$ are complex analytic, the property to have an isolated special point is a condition on the classes of these 1-forms in the module

$$
\Omega_{V, 0}^{1}=\Omega_{\mathbb{C}^{N}, 0}^{1} /\left\{f \cdot \Omega_{\mathbb{C}^{N}, 0}^{1}+d f \cdot \mathcal{O}_{\mathbb{C}^{N}, 0} \mid f \in \mathcal{J}_{V}\right\}
$$

of germs of 1-forms on the variety $V\left(\mathcal{J}_{V}\right.$ is the ideal of germs of functions vanishing on $V$ ).

Remark. For the case $s=1$ (and therefore $k_{1}=n$ ), i.e. for one 1-form $\omega$, we discussed the notion of a singular point of the 1-form $\omega$ on $V$. One can easily see that a special point of the 1-form $\omega$ on $V$ is singular, but not vice versa. (E.g. the origin is a singular point of the 1-form $d x$ on the cone $\left\{x^{2}+y^{2}+z^{2}=0\right\}$, but not a special one.) On a smooth variety these two notions coincide.

Definition: A special (singular) point of a collection $\left\{\omega_{j}^{(i)}\right\}$ of germs of 1 -forms on a smooth $n$-dimensional variety $V$ is non-degenerate if the map $\Psi_{V}: V \cap U \rightarrow \mathcal{M}_{n, \mathbf{k}}$ described above is transversal to $D_{n, \mathbf{k}} \subset \mathcal{M}_{n, \mathbf{k}}$ at a non-singular point of it.

Let

$$
\mathcal{L}_{\mathbf{k}}=\prod_{i=1}^{s} \prod_{j=1}^{n-k_{i}+1} \mathbb{C}_{i j}^{N *}
$$


be the space of collections of linear functions on $\mathbb{C}^{N}$ (i.e. of 1-forms with constant coefficients). The following statement holds.

Proposition 7. There exists an open and dense set $U \subset \mathcal{L}_{\mathbf{k}}$ such that each collection $\left\{\ell_{j}^{(i)}\right\} \in U$ has only isolated special points on $V$ and, moreover, all these points belong to the smooth part $V_{\text {reg }}$ of the variety $V$ and are non-degenerate.

Corollary 2. Let $\left\{\omega_{j}^{(i)}\right\}$ be a collection of 1-forms on $V$ with an isolated special point at the origin. Then there exists a deformation $\left\{\widetilde{\omega}_{j}^{(i)}\right\}$ of the collection $\left\{\omega_{j}^{(i)}\right\}$ whose special points lie in $V_{\text {reg }}$ and are non-degenerate. Moreover, as such a deformation one can use $\left\{\omega_{j}^{(i)}+\lambda \ell_{j}^{(i)}\right\}$ with a generic collection $\left\{\ell_{j}^{(i)}\right\} \in \mathcal{L}_{\mathbf{k}}$.

Corollary 3. The set of collections of holomorphic 1-forms with a nonisolated special point at the origin has infinite codimension in the space of all holomorphic collections.

Let $\left\{\omega_{j}^{(i)}\right\}$ be a collection of germs of 1 -forms on $(V, 0)$ with an isolated special point at the origin. Let $\nu: \widehat{V} \rightarrow V$ be the Nash transformation of the variety $V \subset B_{\varepsilon}(0)$ (see Section 5 ). The collection of 1-forms $\left\{\omega_{j}^{(i)}\right\}$ gives rise to a section $\widehat{\omega}$ of the bundle

$$
\widehat{\mathbb{T}}=\bigoplus_{i=1}^{s} \bigoplus_{j=1}^{n-k_{i}+1} \widehat{T}_{i, j}^{*}
$$

where $\widehat{T}_{i, j}^{*}$ are copies of the dual Nash bundle $\widehat{T}^{*}$ over the Nash transform $\widehat{V}$ numbered by indices $i$ and $j$. Let $\widehat{\mathbb{D}} \subset \widehat{\mathbb{T}}$ be the set of pairs $\left(x,\left\{\alpha_{j}^{(i)}\right\}\right)$ where $x \in \widehat{V}$ and the collection $\left\{\alpha_{j}^{(i)}\right\}$ of elements of $\widehat{T}_{x}^{*}$ (i.e. of linear functions on $\left.\widehat{T}_{x}\right)$ is such that $\alpha_{1}^{(i)}, \ldots, \alpha_{n-k_{i}+1}^{(i)}$ are linearly dependent for each $i=1, \ldots, s$. The image of the section $\widehat{\omega}$ does not intersect $\widehat{\mathbb{D}}$ outside of the preimage $\nu^{-1}(0) \subset \widehat{V}$ of the origin. The map $\widehat{\mathbb{T}} \backslash \widehat{\mathbb{D}} \rightarrow \widehat{V}$ is a fibre bundle. The fibre $W_{x}=\widehat{\mathbb{T}} \backslash \widehat{\mathbb{D}}$ of it is $(2 n-2)$-connected, its homology group $H_{2 n-1}\left(W_{x} ; \mathbb{Z}\right)$ is isomorphic to $\mathbb{Z}$ and has a natural generator (see above). The latter fact implies that the fibre bundle $\widehat{\mathbb{T}} \backslash \widehat{\mathbb{D}} \rightarrow \widehat{V}$ is homotopically simple in dimension $2 n-1$, i.e. the fundamental group $\pi_{1}(\widehat{V})$ of the base acts trivially on the homotopy group $\pi_{2 n-1}\left(W_{x}\right)$ of the fibre, the last one being isomorphic to the homology group $H_{2 n-1}\left(W_{x}\right)$ : see, e.g., St51].

Definition: The local Chern obstruction $\mathrm{Ch}_{V, 0}\left\{\omega_{j}^{(i)}\right\}$ of the collections of germs of 1 -forms $\left\{\omega_{j}^{(i)}\right\}$ on $(V, 0)$ at the origin is the (primary) obstruction to extend the section $\widehat{\omega}$ of the fibre bundle $\widehat{\mathbb{T}} \backslash \widehat{\mathbb{D}} \rightarrow \widehat{V}$ from the preimage of a neighbourhood of the sphere $S_{\varepsilon}=\partial B_{\varepsilon}$ to $\widehat{V}$, more precisely its value 
(as an element of the homology group $H^{2 n}\left(\nu^{-1}\left(V \cap B_{\varepsilon}\right), \nu^{-1}\left(V \cap S_{\varepsilon}\right) ; \mathbb{Z}\right)$ ) on the fundamental class of the pair $\left(\nu^{-1}\left(V \cap B_{\varepsilon}\right), \nu^{-1}\left(V \cap S_{\varepsilon}\right)\right)$.

The definition of the local Chern obstruction $\mathrm{Ch}_{V, 0}\left\{\omega_{j}^{(i)}\right\}$ can be reformulated in the following way. Let $\mathcal{D}_{V}^{\mathrm{k}} \subset \mathbb{C}^{N} \times \mathcal{L}^{\mathrm{k}}$ be the closure of the set of pairs $\left(x,\left\{\ell_{j}^{(i)}\right\}\right)$ such that $x \in V_{\text {reg }}$ and the restrictions of the linear functions $\ell_{1}^{(i)}, \ldots, \ell_{n-k_{i}+1}^{(i)}$ to $T_{x} V_{\text {reg }} \subset \mathbb{C}^{N}$ are linearly dependent for each $i=1, \ldots, s$. (For $s=1, \mathbf{k}=\{n\}, \mathcal{D}_{V}^{\mathbf{k}}$ is the (non-projectivized) conormal space of $V$ Tei82].) The collection $\left\{\omega_{j}^{(i)}\right\}$ of germs of 1 -forms on $\left(\mathbb{C}^{N}, 0\right)$ defines a section $\check{\omega}$ of the trivial fibre bundle $\mathbb{C}^{N} \times \mathcal{L}^{\mathbf{k}} \rightarrow \mathbb{C}^{N}$. Then

$$
\mathrm{Ch}_{V, 0}\left\{\omega_{j}^{(i)}\right\}=\left(\check{\omega}\left(\mathbb{C}^{N}\right) \circ \mathcal{D}_{V}^{\mathbf{k}}\right)_{0}
$$

where $(\cdot \circ \cdot)_{0}$ is the intersection number at the origin in $\mathbb{C}^{N} \times \mathcal{L}^{\mathbf{k}}$. This description can be considered as a generalization of an expression of the local Euler obstruction as a micro-local intersection number defined in KS90, see also [Schü03, Sections 5.0.3 and 5.2.1] and Schü04].

Remark. On a smooth manifold $V$ the local Chern obstruction $\mathrm{Ch}_{V, 0}\left\{\omega_{j}^{(i)}\right\}$ coincides with the index $\operatorname{ind}_{V, 0}\left\{\omega_{j}^{(i)}\right\}$ of the collection $\left\{\omega_{j}^{(i)}\right\}$ defined above.

Remark. The local Euler obstruction is defined for vector fields as well as for 1-forms. One can see that vector fields are not well adapted to a definition of the local Chern obstruction. A more or less direct version of the definition above for vector fields demands to consider vector fields on a singular variety $V \subset \mathbb{C}^{N}$ to be sections $X=X(x)$ of $T \mathbb{C}_{\mid V}^{N}$ such that $X(x) \in T_{x} V \subset T_{x} \mathbb{C}^{N}$ ( $\operatorname{dim} T_{x} V$ is not constant). (Traditionally vector fields tangent to smooth strata of the variety $V$ are considered.) There exist only continuous (non-trivial, i.e. with $s>1$ ) collections of such vector fields "on $V$ " with isolated special points, but not holomorphic ones.

Remark. The definition of the local Chern obstruction $\operatorname{Ch}_{V, 0}\left\{\omega_{j}^{(i)}\right\}$ may also be formulated in terms of a collection $\left\{\omega^{(i)}\right\}$ of germs of 1-forms with values in vector spaces $L_{i}$ of dimensions $n-k_{i}+1$. Therefore (via differentials) it is also defined for a collection $\left\{f^{(i)}\right\}$ of germs of maps $f^{(i)}$ : $\left(\mathbb{C}^{N}, 0\right) \rightarrow\left(\mathbb{C}^{n-k_{i}+1}, 0\right)$ (just as the Euler obstruction is defined for a germ of a function).

Being a (primary) obstruction, the local Chern obstruction satisfies the law of conservation of number, i.e. if a collection of 1 -forms $\left\{\widetilde{\omega}_{j}^{(i)}\right\}$ is a deformation of the collection $\left\{\omega_{j}^{(i)}\right\}$ and has isolated special points on $V$, then

$$
\mathrm{Ch}_{V, 0}\left\{\omega_{j}^{(i)}\right\}=\sum \mathrm{Ch}_{V, Q}\left\{\widetilde{\omega}_{j}^{(i)}\right\}
$$


where the sum on the right hand side is over all special points $Q$ of the collection $\left\{\widetilde{\omega}_{j}^{(i)}\right\}$ on $V$ in a neighbourhood of the origin. With Corollary 2 this implies the following statements.

Proposition 8. The local Chern obstruction $\mathrm{Ch}_{V, 0}\left\{\omega_{j}^{(i)}\right\}$ of a collection $\left\{\omega_{j}^{(i)}\right\}$ of germs of holomorphic 1-forms is equal to the number of special points on $V$ of a generic (holomorphic) deformation of the collection.

This statement is an analogue of Proposition 2.3 in STV05b.

Proposition 9. If a collection $\left\{\omega_{j}^{(i)}\right\}$ of 1-forms on a compact (say, projective) variety $V$ has only isolated special points, then the sum of local Chern obstructions of the collection $\left\{\omega_{j}^{(i)}\right\}$ at these points does not depend on the collection and therefore is an invariant of the variety.

It is reasonable to consider this sum as $\left((-1)^{n}\right.$ times $)$ the corresponding Chern number of the singular variety $V$.

Let $(V, 0)$ be an isolated complete intersection singularity. The fact that both the Chern obstruction and the index of a collection $\left\{\omega_{j}^{(i)}\right\}$ of 1-forms satisfy the law of conservation of number and they coincide on a smooth manifold yields the following statement.

Proposition 10. For a collection $\left\{\omega_{j}^{(i)}\right\}$ on an isolated complete intersection singularity $(V, 0)$ the difference

$$
\operatorname{ind}_{V, 0}\left\{\omega_{j}^{(i)}\right\}-\mathrm{Ch}_{V, 0}\left\{\omega_{j}^{(i)}\right\}
$$

does not depend on the collection and therefore is an invariant of the germ of the variety.

Since by Proposition $9 \mathrm{Ch}_{V, 0}\left\{\ell_{j}^{(i)}\right\}=0$ for a generic collection $\left\{\ell_{j}^{(i)}\right\}$ of linear functions on $\mathbb{C}^{N}$, one has the following statement.

Corollary 4. One has

$$
\mathrm{Ch}_{V, 0}\left\{\omega_{j}^{(i)}\right\}=\operatorname{ind}_{V, 0}\left\{\omega_{j}^{(i)}\right\}-\operatorname{ind}_{V, 0}\left\{\ell_{j}^{(i)}\right\}
$$

for a generic collection $\left\{\ell_{j}^{(i)}\right\}$ of linear functions on $\mathbb{C}^{N}$.

\section{REFERENCES}

[ASV98] Aguilar, M. A., Seade, J. A., Verjovsky, A.: Indices of vector fields and topological invariants of real analytic singularities. J. reine angew. Math. 504, 159-176 (1998)

[Ar78] Arnold, V. I.: Index of a singular point of a vector field, the PetrovskiiOleinik inequality, and mixed Hodge structures. Funct. Anal. Appl. 12, no.1, 1-12 (1978))

[Ar79] Arnold, V. I.: Indices of singular points of 1-forms on a manifold with boundary, convolution of invariants of reflection groups, and singular projections of smooth surfaces. Russian Math. Surveys 34:2, 1-42 (1979) 
[AGV85] Arnold, V. I., Gusein-Zade, S. M., Varchenko, A. N.: Singularities of Differentiable Maps, Vol. I, Birkhäuser, Boston Basel Berlin (1985)

[Ar04] Arnold, V. I. (ed.): Arnold's Problems. Springer-Verlag, Berlin; PHASIS, Moscow (2004)

[BB70] Baum, P. F., Bott, R.: On the zeros of a meromorphic vector field. In: Essays on Topology and Related Topics (Mémoires dédiés à Georges de Rham), Springer, New York (1970), pp.29-47

[BG94] Bonatti, Ch., Gómez-Mont, X.: The index of holomorphic vector fields on singular varieties I. Astérisque 222, 9-35 (1994)

[BEG05] Bothmer, H.-Ch. Graf von, Ebeling, W., Gómez-Mont, X: The homological index of a vector field on an isolated complete intersection singularity. Preprint 2005.

[Br00] Brasselet, J.-P.: From Chern classes to Milnor classes - a history of characteristic classes for singular varieties. In: Brasselet, J.-P., Suwa, T. (eds) Singularities-Sapporo 1998, Adv. Stud. Pure Math. 29, Kinokuniya, Tokyo (2000), pp. 31-52

[BLS00] Brasselet, J.-P., Lê D. T., Seade, J.: Euler obstruction and indices of vector fields. Topology 39, 1193-1208 (2000)

[BMPS04] Brasselet, J.-P., Massey, D., Parameswaran, A. J., Seade, J.: Euler obstruction and defects of functions on singular varieties. J. London Math. Soc. (2) 70, 59-76 (2004)

[BSS05a] Brasselet, J.-P., Seade, J., Suwa, T.: Proportionality of indices of 1-forms on singular varieties. math.AG/0503428 to appear in the Proceedings of the Third Franco-Japanese Symposium on Singularities

[BSS05b] Brasselet, J.-P., Seade, J., Suwa, T.: A proof of the proportionality theorem. math.AG/0511601

[BS81] Brasselet, J.-P., Schwartz, M.-H.: Sur les classes de Chern d'un ensemble analytique complexe. In: Caractéristique d'Euler-Poincaré, Astérisque 8283, 93-147 (1981)

[BG80] Buchweitz, R.-O., Greuel, G.-M.: The Milnor number and deformations of complex curve singularities. Invent. Math. 58, 241-281 (1980)

[EG99] Ebeling, W., Gusein-Zade, S. M.: On the index of a vector field at an isolated singularity. In: Bierstone, E., Khesin, B., Khovanskii, A., Marsden, J. (eds) The Arnoldfest: Proceedings of a Conference in Honour of V. I. Arnold for his Sixtieth Birthday. Fields Institute Communications, Vol. 24, Am. Math. Soc., Providence (1999), pp. 141-152.

[EG01] Ebeling, W., Gusein-Zade, S. M.: On the index of a holomorphic 1-form on an isolated complete intersection singularity. Russian Math. Doklady, 380, no.4, 458-461 (2001)

[EG03a] Ebeling, W., Gusein-Zade, S. M.: Indices of 1-forms on an isolated complete intersection singularity. Moscow Math. J. 3, 439-455 (2003)

[EG03b] Ebeling, W., Gusein-Zade, S. M.: On the indices of 1-forms on isolated singularities of complete intersections. In: Fundamental mathematics today. Independent University of Moscow, Moscow (2003), pp. 110-134 (in Russian)

[EG04] Ebeling, W., Gusein-Zade, S. M.: On indices of meromorphic 1-forms. Compos. Math. 140 809-817 (2004)

[EG05a] Ebeling, W., Gusein-Zade, S. M.: Radial index and Euler obstruction of a 1-form on a singular variety. Geom. Dedicata 113, 231-241 (2005)

[EG05b] Ebeling, W., Gusein-Zade, S. M.: Indices of vector fields or 1-forms and characteristic numbers. Bull. London Math. Soc. 37, 747-754 (2005)

[EG05c] Ebeling, W., Gusein-Zade, S. M.: Quadratic forms for a 1-form on an isolated complete intersection singularity. math.AG/0503336 Math. Z. (to appear) 
[EG05d] Ebeling, W., Gusein-Zade, S. M.: Chern obstructions for collections of 1forms on singular varieties. math.AG/0503422

[EGS04] Ebeling, W., Gusein-Zade, S. M., Seade, J.: Homological index for 1-forms and a Milnor number for isolated singularities. Int. J. of Math. 15, 895-905 (2004)

[EL77] Eisenbud, D., Levine, H.: An algebraic formula for the degree of a $C^{\infty}$ map germ. Ann. Math. 106, 19-38 (1977)

[Es05a] Esterov, A.: Indices of 1-forms and Newton polyhedra. Rev. Mat. Complut. 18, no. 1, 233-242 (2005)

[Es05b] Esterov, A.: Indices of 1-forms, resultants and Newton polyhedra. Russian Math. Surveys 60:2, 352-353 (2005)

[Ga05] Gaffney, T.: The multiplicity polar theorem and isolated singularities. math.AG/0509285

[GG02] Giraldo, L., Gómez-Mont, X.: A law of conservation of number for local Euler characteristics. Contemp. Math. 311, 251-259 (2002)

[GGM02] Giraldo, L., Gómez-Mont, X., Mardešić, P.: On the index of vector fields tangent to hypersurfaces with non-isolated singularities. J. London Math. Soc. (2) 65, no. 2, 418-438 (2002)

[Góm98] Gómez-Mont, X.: An algebraic formula for the index of a vector field on a hypersurface with an isolated singularity. J. Alg. Geom. 7, 731-752 (1998)

[GM97] Gómez-Mont, X., Mardešić, P.: The index of a vector field tangent to a hypersurface and the signature of the relative Jacobian determinant. Ann. Inst. Fourier 47, 1523-1539 (1997)

[GM99] Gómez-Mont, X., Mardešić, P.: The index of a vector field tangent to an odddimensional hypersurface and the signature of the relative Hessian. Funct. Anal. Appl. 33, no.1, 1-10 (1999)

[GSV91] Gómez-Mont, X., Seade, J., Verjovsky, A.: The index of a holomorphic flow with an isolated singularity. Math. Ann. 291, 737-751 (1991)

[Gor00] Goryunov, V.: Functions on space curves. J. London Math. Soc. 61, 807-822 (2000)

[Gr75] Greuel, G.-M.: Der Gauß-Manin-Zusammenhang isolierter Singularitäten von vollständigen Durchschnitten. Math. Ann. 214, 235-266 (1975)

[GH78] Griffiths, Ph., Harris, J.: Principles of Algebraic Geometry. John Wiley \& Sons, New York etc. (1978)

[Gu84] Gusein-Zade, S. M.: The index of a singular point of a gradient vector field. Funct. Anal. Appl. 18, no.1, 6-10 (1984)

[HS98] Honda, T., Suwa, T.: Residue formulas for meromorphic functions on surfaces. Ann. Fac. Sci. Toulouse Math. (6) 7, 443-463 (1998)

[Hu75] Husemoller, D.: Fibre Bundles. Second Edition. Graduate Texts in Math. 20, Springer-Verlag, New York Heidelberg Berlin (1975)

[IS03] Izawa, T., Suwa, T.: Multiplicity of functions on singular varieties. Intern. J. Math. 14, 541-558 (2003)

[KS90] Kashiwara, M., Schapira, P.: Sheaves on Manifolds. Springer-Verlag, Berlin Heidelberg New York (1990)

[Kh77] Khimshiashvili, G. N.: On the local degree of a smooth map. Comm. Acad. Sci. Georgian SSR. 85, no.2, 309-311 (1977) (in Russian)

[KT99] King, H., Trotman, D.: Poincaré-Hopf theorems on singular spaces. Preprint (1999)

[Kl02] Klehn, O.: Local residues of holomorphic 1-forms on an isolated surface singularity. Manuscripta Math. 109, no. 1, 93-108 (2002)

[Kl03] Klehn, O.: On the index of a vector field tangent to a hypersurface with non-isolated zero in the embedding space. Math. Nachr. 260, 48-57 (2003) 
[K105] Klehn, O.: Real and complex indices of vector fields on complete intersection curves with isolated singularity. Compos. Math. 141, no. 2, 525-540 (2005)

[Lê74] Lê, D. T.: Computation of the Milnor number of an isolated singularity of a complete intersection. Funct. Anal. Appl. 8, 127-131 (1974)

[Lê76] Lê, D. T.: Some remarks on relative monodromy. In: Holm, P. (ed) Real and Complex Singularities, Oslo 1976. Sijthoff \& Noordhoff, Alphen a.d. Rijn (1977), pp.397-403

[LT81] Lê, D. T., Teissier, B.: Variétés polaires locales et classes de Chern des variétés singulières. Ann. Math. 114, 457-491 (1981)

[LS95] Lehmann, D., Suwa, T.: Residues of holomorphic vector fields relative to singular invariant subvarieties. J. Differential Geom. 42, no. 1, 165-192 (1995)

[LSS95] Lehmann, D., Soares, M., Suwa, T.: On the index of a holomorphic vector field tangent to a singular variety. Bol. Soc. Brasil. Mat. (N.S.) 26, no. 2, 183-199 (1995)

[Lo84] Looijenga, E. J. N.: Isolated Singular Points on Complete Intersections. London Math. Soc. Lecture Note Series 77, Cambridge University Press, Cambridge (1984)

[Mac74] MacPherson, R.: Chern classes for singular varieties. Annals of Math. 100, 423-432 (1974)

[MvS01] Mond, D., van Straten, D.: Milnor number equals Tjurina number for functions on space curves. J. London Math. Soc. 63, 177-187 (2001)

[MvS90] Montaldi, J., van Straten, D.: One-forms on singular curves and the topology of real curve singularities. Topology 29, 501-510 (1990)

[Pa67] Palamodov, V. P.: The multiplicity of a holomorphic transformation. Funktsional. Anal. i Prilozhen. 1:3, 54-65 (1967) (Engl. translation in Funct. Anal. Appl.

[Pi74] Pinkham, H. C.: Deformations of Algebraic Varieties with $G_{m}$ Action. Astérisque 20 (1974)

[Schü03] Schürmann, J.: Topology of Singular Spaces and Constructible Sheaves. Birkhäuser, Basel (2003)

[Schü04] Schürmann, J.: A general intersection formula for Lagrangian cycles. Compositio Math. 140, 1037-1052 (2004)

[Schw65] Schwartz, M.-H.: Classes caractéristiques définies par une stratification d'une variété analytique complexe. C. R. Acad. Sci. Paris Sér. I Math. 260, 3262-3264, 3535-3537 (1965)

[Schw86a] Schwartz, M.-H.: Champs radiaux et préradiaux associés à une stratification. C. R. Acad. Sci. Paris Sér. I Math. 303, 239-241 (1986)

[Schw86b] Schwartz, M.-H.: Une généralisation du théorème de Hopf pour les champs sortants. C. R. Acad. Sci. Paris Sér. I Math. 303, 307-309 (1986)

[Schw91] Schwartz, M.-H.: Champs radiaux sur une stratification analytique. Travaux en Cours, 39, Hermann, Paris (1991)

[Se87] Seade, J.: The index of a vector field on a complex analytic surface with singularities. The Lefschetz centennial conference, Part III (Mexico City, 1984). Contemp. Math., 58, III, Amer. Math. Soc., Providence, RI (1987), pp. 225-232

[Se95] Seade, J.: The index of a vector field on a singular variety. In: Lê, D. T., Saito, K., Teissier, B. (eds) Singularity theory (Trieste, 1991). World Sci. Publishing, River Edge, NJ (1995), pp. 673-696

[Se02] Seade, J.: Indices of vector fields and Chern classes for singular varieties. In: Bridson, M. R., Salamon, S. S. (eds) Invitations to geometry and topology. Oxf. Grad. Texts Math., 7, Oxford Univ. Press, Oxford (2002), pp. 292-320

[SS96] Seade, J. A., Suwa, T.: A residue formula for the index of a holomorphic flow. Math. Ann. 304, 621-634 (1996) 
[SS98] Seade, J., Suwa, T.: An adjunction formula for local complete intersections. Intern. J. Math. 9, 759-768 (1998)

[STV05a] Seade, J., Tibăr, M., Verjovsky, A.: Global Euler obstruction and polar invariants. Math. Ann. 333, 393-403 (2005)

[STV05b] Seade, J., Tibăr, M., Verjovsky, A.: Milnor numbers and Euler obstruction. Bull. Braz. Math. Soc. (N.S.) 36, no. 2, 275-283 (2005)

[St51] Steenrod, N.: The Topology of Fibre Bundles. Princeton Math. Series, Vol. 14, Princeton University Press, Princeton, N. J. (1951)

[Su95] Suwa, T.: Indices of holomorphic vector fields relative to invariant curves on surfaces. Proc. Amer. Math. Soc. 123, no. 10, 2989-2997 (1995)

[Su98] Suwa, T.: Indices of vector fields and residues of singular holomorphic foliations. Actualités Mathématiques. Hermann, Paris (1998)

[Su02] Suwa, T.: Residues of Chern classes on singular varieties. In: Brasselet, J.-P., Suwa, T. (eds) Singularités Franco-Japonaises. Séminaires et Congrès 10, Soc. Math. France, Paris (2005), pp. 265-286

[Tei82] Teissier, B.: Variétés polaires. II. Multiplicités polaires, sections planes, et conditions de Whitney. In: Aroca, J. M., Buchweitz, R., Giusti, M., Merle, M. (eds) Algebraic geometry (La Rábida, 1981). Lecture Notes in Math., Vol. 961, Springer, Berlin (1982), pp. 314-491

[Va85] Varchenko, A. N.: Local residue and the intersection form in vanishing cohomology. Math. USSR Izv. 26, 31-52 (1986)

Universität Hannover, Institut für Algebraische Geometrie, Postfach 6009, D-30060 Hannover, Germany

E-mail address: ebeling@math.uni-hannover.de

Moscow State University, Faculty of Mechanics and Mathematics, Moscow, 119992, RUSSIA

E-mail address: sabir@mccme.ru 